Check for updates

Cite this: RSC Adv., 2020, 10, 35109

Received 30th July 2020

Accepted 15th September 2020

DOI: $10.1039 /$ dOra07472a

rsc.li/rsc-advances

\section{Formation of trisubstituted buta-1,3-dienes and $\alpha, \beta$-unsaturated ketones via the reaction of functionalized vinyl phosphates and vinyl phosphordiamidates with organometallic reagents $\dagger$}

\begin{abstract}
Petr Oeser, +ła Jakub Koudelka, ła Hana Dvořákováb and Tomáš Tobrman (iD *a
We studied the reactions of vinyl phosphates and vinyl phosphordiamidates containing an ester functional group with organometallic reagents. We found that the functionalized vinyl phosphates were smoothly converted into tri- and tetrasubstituted buta-1,3-dienes via the reaction with aryllithium reagents. Moreover, the vinyl phosphordiamidates were converted into $\alpha, \beta$-unsaturated ketones using Grignard reagents. Based on the performed experiments, we proposed a reaction mechanism, which was confirmed by means of the isolation of key intermediates.
\end{abstract}

\section{Introduction}

A Kumada-Tamao-Corriu (KTC) reaction is a representative example of the traditional cross-coupling reaction of an electrophilic template with an organometallic reagent, as represented by Grignard reagents. This reaction is frequently used for the formation of a C-C bond. ${ }^{1}$ Among the various electrophilic templates that are suitable for use in a KTC reaction, substrates containing an activated $\mathrm{C}-\mathrm{O}$ bond are considered particularly attractive. The reason behind the popularity of such substrates is their availability, which is associated with their preparation. Recent examples of the cross-coupling reactions of ethers, ${ }^{2}$ tosylates, ${ }^{3}$ and triflates ${ }^{4}$ with Grignard reagents all illustrate the KTC reaction potential of compounds with an activated C-O bond. Using this approach, a wide variety of substances can be prepared, including substituted alkenes.

The stereoselective synthesis of di-, tri-, ${ }^{5}$ and tetrasubstituted $^{6}$ double bonds has been the subject of significant research attention in recent decades. Vinyl phosphates also play a key role in the synthesis of substituted alkenes. Unsurprisingly, a number of KTC reactions have been described in which the reaction of the vinyl phosphates has been catalyzed by iron, ${ }^{7}$ nickel, ${ }^{8}$ or palladium $^{9}$ catalysts in order to prepare the substituted double bond.

a Department of Organic Chemistry, University of Chemistry and Technology, Prague, Technická 5, 16628 Prague 6, Czech Republic. E-mail: tomas.tobrman@vscht.cz

${ }^{b}$ Laboratory of NMR Spectroscopy, University of Chemistry and Technology, Prague, Technická 5, 16628 Prague 6, Czech Republic

$\dagger$ Electronic supplementary information (ESI) available. See DOI: 10.1039/d0ra07472a

$\$$ These authors contributed equally to the work.
Despite considerable progress having been made in relation to the preparation and application of functionalized Grignard reagents,$^{10}$ the preparation of alkenes with functional groups that react with Grignard reagents using the KTC reaction remains difficult (Scheme 1). The vinyl phosphates 1 with a carbonyl group attached to the vinyl unit have been used in the stoichiometric synthesis of organoselenium ${ }^{11}$ and organotellurium compounds. ${ }^{12}$ A methodology for the stoichiometric

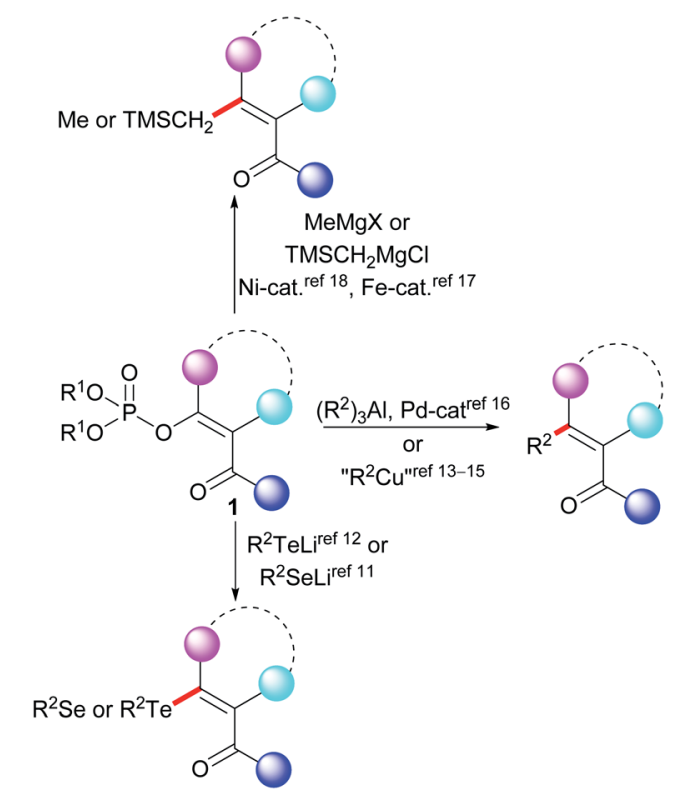

Scheme 1 General scheme representing the replacement of the phosphate group of the functionalized vinyl phosphates 1 . 
cross-coupling reaction of the functionalized vinyl phosphates with dialkylcuprates has also been described. ${ }^{13}$ This methodology has been successfully used in the field of organic synthesis ${ }^{\mathbf{1 4}}$ as well as in the total synthesis of naturally occurring compounds. ${ }^{15}$ The catalytic reactions of the functionalized vinyl phosphates represented by the general structure $\mathbf{1}$ include reactions with organoaluminum compounds, ${ }^{16}$ iron-catalyzed reactions with methylmagnesium halides, ${ }^{17}$ and nickelcatalyzed cross-coupling reactions with trimethylsilylmethylmagnesium chloride. ${ }^{18}$ These prior results contrast with a recent report of the KTC reaction of functionalized vinyl tosylates being performed in dry acetonitrile at $45{ }^{\circ} \mathrm{C} .{ }^{3 b}$ The absence of a general methodology for the KTC reaction of the functionalized vinyl phosphates $\mathbf{1}$ with Grignard reagents may indicate that this type of vinyl phosphate exhibits low stability in the presence of Grignard reagents. Our review of the literature indicated that a systematic study of the reactivity of the functionalized vinyl phosphates $\mathbf{1}$ with organometallic reagents has not previously been reported. Thus, based on our earlier studies of the cross-coupling reactions of vinyl phosphates, ${ }^{19} \mathrm{we}$ decided to investigate the stability of the functionalized vinyl phosphates $\mathbf{2 a - 2 c}$ in the presence of Grignard reagents.

\section{Results and discussion}

We began our study by synthesizing the vinyl phosphate 2 a by means of the enolization of ethyl acetoacetate, followed by the reaction of the formed enolate with diethyl chlorophosphate. The prepared phosphate 2a was mixed with phenylmagnesium chloride, and then the reaction mixture was stirred for 2 hours at $23{ }^{\circ} \mathrm{C}$. After the reaction mixture was quenched with aqueous ammonium chloride, the formation of a complex mixture of products was observed based on analysis of the ${ }^{1} \mathrm{H}$ nuclear magnetic resonance $\left({ }^{1} \mathrm{H}\right.$ NMR) spectrum of the mixture. However, we isolated the trisubstituted diene 3a as a pure compound in an isolated yield of $27 \%$. This finding was particularly interesting, as the synthesis of trisubstituted dienes is generally limited. The palladium-catalyzed coupling of tosylhydrazones with aryl halides, ${ }^{20}$ the selective arylation of vinylarenes, ${ }^{21}$ and diethyl-phosphite-promoted carbonyl olefination $^{22}$ are recently reported methods for the synthesis of substituted buta-1,3-dienes. We observed that the isolated yield of the diene 3a strongly depended on the reaction workup, with comparatively higher yields of the alkene $\mathbf{3 a}$ being achieved with hydrochloric acid, sulfuric acid, and phosphoric acid (Table 1, entries 3-5). The isolated yield of the alkene 3a did not affect the increased equivalents of phenylmagnesium chloride or the use of the diphenylphosphate $\mathbf{2 b}$ (Table 1, entries 6 and 7). The best isolated yield of the diene $\mathbf{3 a}$ was obtained when phenyllithium was used in combination with the phenyl ester 2c (Table 1, entry 9).

Optimized reaction conditions were used to evaluate the scope of the reaction (Scheme 2). The diethyl phosphate 2c reacted with the aryllithium reagents, thereby affording the corresponding dienes $\mathbf{3 a - 3 d}$ in a single step and in good isolated yields. 2-Methoxyphenyllithium, as an example of an ortho-substituted phenyl, gave the product $3 \mathbf{e}$ in a high isolated
Table 1 Optimization of the reaction of the vinyl phosphates $2 a-c$ with phenylmagnesium chloride and phenyllithium

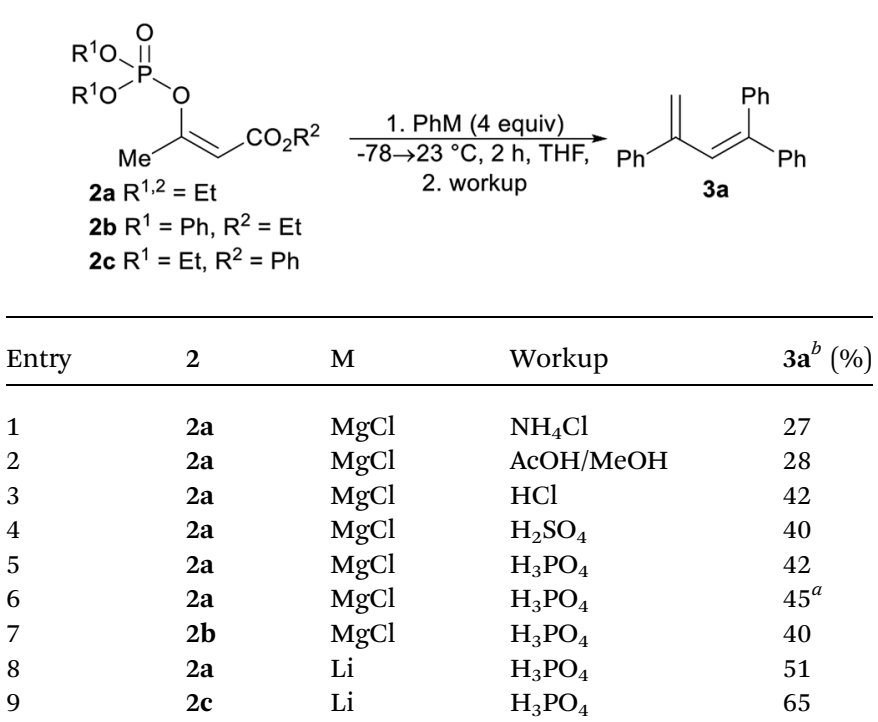

${ }^{a}$ Six equivalents of phenylmagnesium chloride were used. ${ }^{b}$ Isolated yield.

yield. A similar reactivity pattern was observed with regard to the diethyl phosphate with an extended alkyl chain 2d. In this case, the dienes $\mathbf{3 f}-\mathbf{3 i}$ were obtained in similar isolated yields to the dienes $3 \mathbf{a}-3 \mathbf{c}$. The phosphate $\mathbf{2 d}$ was also reacted with 2 methoxyphenyllithium and 2-thienyllithium, as an example of a heteroaryl reagent, in order to give the dienes $\mathbf{3 j}$ and $\mathbf{3 k}$. The
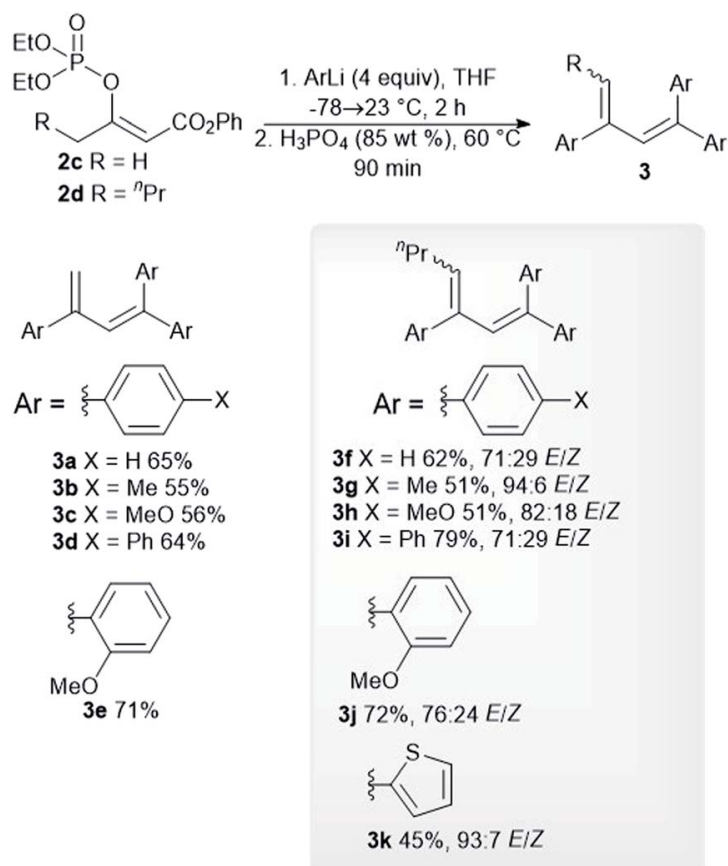

Scheme 2 Reaction of the phosphates $2 \mathrm{c}$ and $2 \mathrm{~d}$ with aryllithium reagents. 


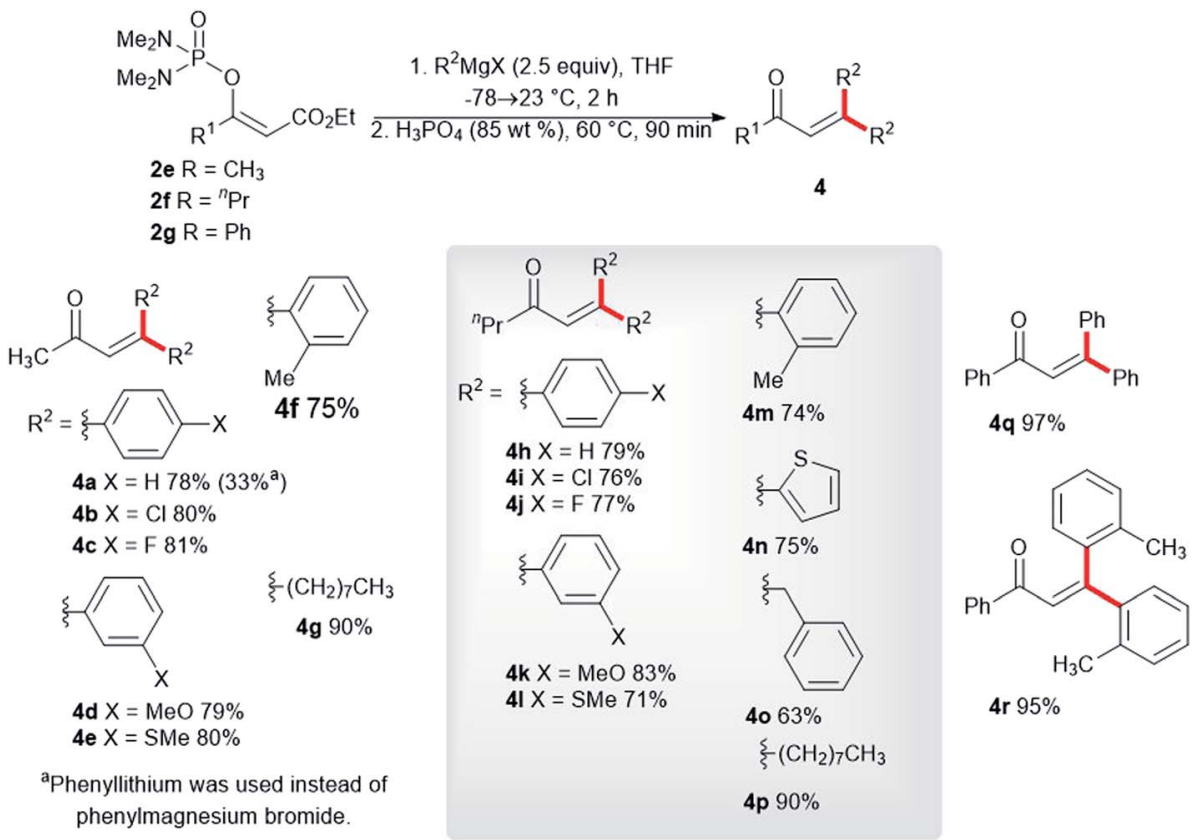

Scheme 3 Synthesis of trisubstituted $\alpha, \beta$-unsaturated ketones via the reaction of the phosphordiamidates $2 \mathrm{e}-2 \mathrm{~g}$ with Grignard reagents.

dienes $3 \mathbf{f}-3 \mathbf{k}$ were obtained as an inseparable mixture of the $(E)$ and $(Z)$-stereoisomers and the double-bond geometry was determined by means of nuclear Overhauser effect (NOE) experiments. The use of an alkyllithium reagent, for example, $n$ butyllithium, failed to give the expected product. Instead, a complex reaction mixture was formed, as observed via the ${ }^{1} \mathrm{H}$ NMR spectroscopy of the crude reaction mixture. It is worth noting that all the dienes $\mathbf{3 a - 3 e}$ were stored in a freezer, as significant decomposition was observed when they were stored at room temperature. However, the tetrasubstituted dienes $\mathbf{3 f}-$ 3k showed excellent long-term storage stability.

We synthesized the vinyl phosphordiamidates $2 \mathbf{e}-2 \mathbf{g}$ so as to test their behavior during the developed one-pot synthesis of the substituted buta-1,3-dienes 3. Surprisingly, the vinyl phosphate $\mathbf{2 e}$ reacted smoothly with phenylmagnesium chloride to give the $\alpha, \beta$-unsaturated ketone 4a in a high isolated yield (Scheme 3). $\alpha, \beta$-Unsaturated ketones are known for their medicinal applications, ${ }^{23}$ and they are also valuable building blocks in relation to organic synthesis. ${ }^{24}$ Therefore, significant research efforts have been dedicated to identifying a novel approach to conjugated enones. ${ }^{25}$ Nickel-catalyzed 1,2-acyl migration, ${ }^{26}$ nucleophilic addition to enones, ${ }^{27}$ the crosscoupling reaction between acid fluorides and vinyl triflates, ${ }^{28}$ and carbonyl group olefinations ${ }^{29}$ are examples of such reactions. The conversion of the vinyl phosphordiamidate $2 \mathbf{e}$ into the ketone 4a observed in our study represents a hitherto undescribed reaction of phosphordiamidate en route to becoming $\alpha, \beta$-unsaturated ketones. Further investigation in this regard showed that a significantly lower isolated yield of the ketone $\mathbf{4 a}$ was obtained with phenyllithium. Uniformly high yields of the corresponding ketones were obtained with the other para-, meta-, and ortho-substituted phenylmagnesium halides $\mathbf{4 b}-\mathbf{4 f}$. Almost the same isolated yields of the ketones 4h-4m were obtained for the vinyl phosphordiamidate 2 f. Additionally, the 2-thienylmagnesium bromide 4n, benzylmagnesium chloride $\mathbf{4 o}$, and octylmagnesium chloride $\mathbf{4 p}$ reacted well with the phosphordiamidate $\mathbf{2 f}$ under the tested reaction conditions. The examination of the reactivity of the variously substituted vinyl phosphordiamidates was also extended to a phosphordiamidate with a phenyl substituent rather than an alkyl group, and the alkenes $\mathbf{4 q}$ and $\mathbf{4 r}$ were obtained in quantitative isolated yields.

Table 2 Reaction of the cyclic vinyl phosphordiamidate $2 \mathrm{~h}$ with phenyllithium and phenylmagnesium chloride

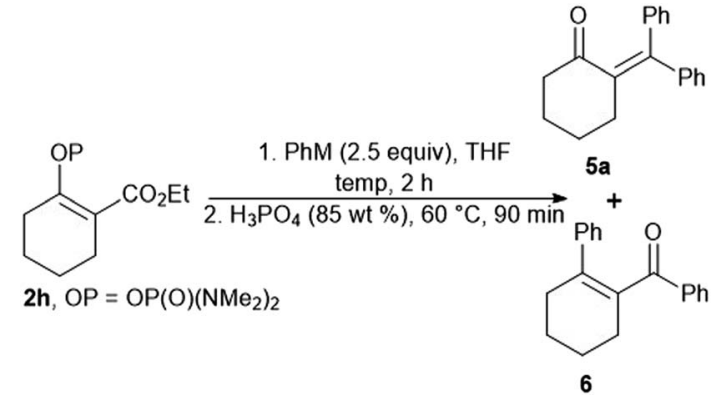

\begin{tabular}{lllll}
\hline Entry & $\mathbf{M}$ & Temp. $\left[{ }^{\circ} \mathrm{C}\right]$ & $\mathbf{5 a}^{a}[\%]$ & $\mathbf{6}^{a}[\%]$ \\
\hline 1 & $\mathrm{MgCl}$ & -78 to 23 & 36 & 33 \\
2 & $\mathrm{MgCl}$ & -40 & 24 & 8 \\
3 & $\mathrm{MgCl}^{b}$ & 0 to 23 & 25 & 45 \\
4 & $\mathrm{Li}$ & -78 to 23 & 67 & 0
\end{tabular}

${ }^{a}$ Isolated yield. ${ }^{b} 10 \mathrm{~mol} \%$ of CuI was used as a catalyst. 


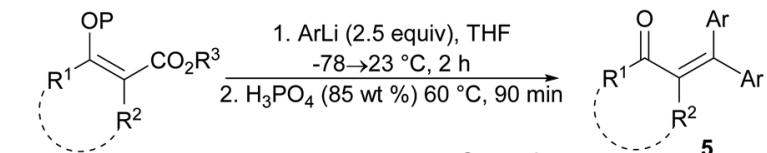

2i $\mathrm{R}^{1,2}=\left(\mathrm{CH}_{2}\right)_{3}, \mathrm{R}^{3}=\mathrm{Me}$

2j $\mathrm{R}^{1,2}=\left(\mathrm{CH}_{2}\right)_{5}, \mathrm{R}^{3}=\mathrm{Me}$

2k $\mathbf{R}^{1,2}=\mathrm{CH}_{3}, \mathbf{R}^{3}=\mathrm{Et}$

$\mathrm{OP}=\mathrm{OP}(\mathrm{O})\left(\mathrm{NMe}_{2}\right)_{2}$

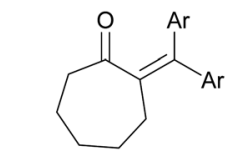

5d $\mathrm{Ar}=\mathrm{Ph}, 83 \%$

$5 \mathrm{e} \mathrm{Ar}=4-\mathrm{MeOC}_{6} \mathrm{H}_{4}, 90 \%$

5f $\mathrm{Ar}=2$-thienyl, $40 \%$

Scheme 4 Synthesis of tetrasubstituted $\alpha, \beta$-unsaturated ketones via the reaction of the phosphates $2 \mathrm{i}-2 \mathrm{k}$ with organolithium reagents

Our attempts to prepare enones with a tetrasubstituted double bond by means of the reaction of the vinyl phosphordiamidate $\mathbf{2 h}$ with phenylmagnesium chloride proved to be less effective, with the formation of the ketones $5 \mathbf{a}$ and $\mathbf{6}$ being observed (Table 2, entry 1 ). Therefore, we tried to optimize the studied reaction in order to obtain only the ketone 5a. However, the use of phenylmagnesium chloride always gave a mixture of the ketones $\mathbf{5 a}$ and $\mathbf{6}$, regardless of the reaction conditions (Table 2, entries 2 and 3). The desired outcome was ultimately achieved through the reaction between phenyllithium and the phosphordiamidate $\mathbf{2 h}$, and the ketone $\mathbf{5 a}$ was obtained in an isolated yield of $67 \%$ (Table 2 , entry 4 ).

The extension of the studied reaction to the cyclic vinyl phosphordiamidates $2 \mathbf{i}$ and $2 \mathbf{j}$ was successful in terms of the preparation of substituted cyclopentanones and cycloheptanones. In both cases, the expected ketones $\mathbf{5 b}, \mathbf{5 c}, \mathbf{5 d}$, and $\mathbf{5 e}$ were obtained in very good isolated yields. Similar results were obtained for the acyclic phosphordiamidate $\mathbf{2 k}$. Only the use of 2-thienyllithium proved less effective, and the reaction products $5 \mathbf{f}$ and $5 \mathbf{i}$ were obtained in low isolated yields (Scheme 4).

As a result of the performed experiments, we were able to propose a mechanism for the observed transformations. We assumed that during the first step, an organometallic reagent addition to the ester functional group occurred and formed a common intermediate 7 . The intermediate 7 with a phosphate group subsequently underwent trans-phosphorylation, with the resultant elimination forming an unsaturated ketone $\mathbf{4 a}$, which was obtained in a low isolated yield of $35 \%$ when the crude reaction mixture was quenched at $-78{ }^{\circ} \mathrm{C}$. The resulting ketone 4a reacted with an organolithium reagent to form the corresponding tertiary alcohol, which was then dehydrated by phosphoric acid to give the diene 3a. This hypothesis was confirmed by the reaction of the ketone $4 \mathbf{a}$ with phenyllithium, with the subsequent dehydration giving the diene $3 \mathbf{a}$ in a $93 \%$ isolated yield. We also verified that ethyl acetoacetate did not provide the ketone $\mathbf{4 a}$ or the diene $\mathbf{3 a}$ under the studied conditions. In the case of the intermediate 7 with phosphordiamidate moiety, the trans-phosphorylation reaction is

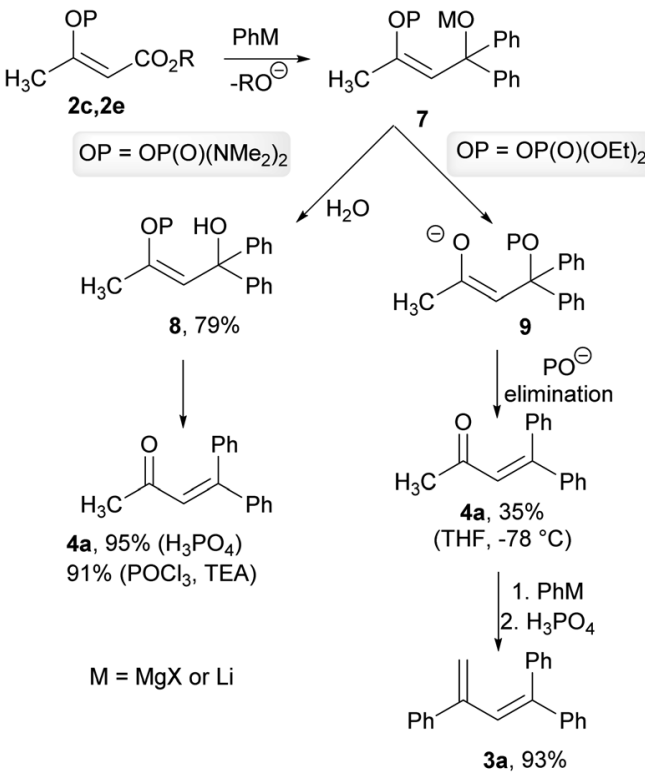

Scheme 5 Proposed mechanism for the reaction of the vinyl phosphate $2 c$ and the vinyl phosphordiamidate $2 e$ with organometallic reagents.

sluggish and the intermediate 7 was converted into the tertiary alcohol 8. The formation of this intermediate was confirmed by means of the hydrolysis of the crude reaction mixture with water, and the corresponding alcohol 8 was obtained in a $79 \%$ isolated yield. The formation of the ketone $\mathbf{4 a}$ was completed by the alcohol 8 dehydration. This was again confirmed by the dehydration of the independently prepared alcohol 8 into the product $\mathbf{4 a}$ in a quantitative isolated yield. Alternatively, the dehydration of the alcohol 8 could be performed using phosphoryl chloride in the presence of triethylamine in a similar isolated yield (Scheme 5).

\section{Conclusion}

In conclusion, we studied the reactivity of both vinyl phosphates and vinyl phosphordiamidates. We found that vinyl phosphates can be converted into tri- and tetrasubstituted buta-1,3-dienes via the reaction with aryllithium reagents in dry THF at $-78{ }^{\circ} \mathrm{C}$ to $23{ }^{\circ} \mathrm{C}$. The tetrasubstituted dienes were isolated as a mixture of the $(E)$ - and $(Z)$-stereoisomers. In the case of the vinyl phosphordiamidates, the reaction with Grignard reagents under the same reaction conditions resulted in the formation of unsaturated ketones, which were isolated in yields ranging from $24 \%$ to $97 \%$. Based on the experimental results, we were able to propose a mechanism explaining the origins of both products.

\section{Experimental section}

\section{Materials and methods}

All reactions were performed under argon atmosphere. NMR spectra were measured on Varian MercuryPlus $300\left({ }^{1} \mathrm{H}, 300.13\right.$ $\left.\mathrm{MHz} ;{ }^{13} \mathrm{C}, 75.46 \mathrm{MHz}\right)$, Agilent 400 MR DD2 $\left({ }^{1} \mathrm{H}, 400.13 \mathrm{MHz}\right.$; $\left.{ }^{13} \mathrm{C}, 100.61 \mathrm{MHz}\right)$ or Bruker Avance III $500\left({ }^{31} \mathrm{P}, 202.45 \mathrm{MHz}\right)$ 
spectrometer at $298 \mathrm{~K}$. Chemical shifts of 31P NMR spectra are referenced to the signal of $85 \% \mathrm{H}_{3} \mathrm{PO}_{4}$ that was assigned the chemical shift of 0 . Mass spectra were measured on ZAB-SEQ (VG Analytical). The dry and degassed THF was prepared by PureSolv MD7. Silica gel (Merck, Silica Gel 60, 40-63 $\mu \mathrm{m}$ or Merck Silica Gel 60, 63-200 $\mu \mathrm{m}$ ) was used for column chromatography. A phosphate 2 a was prepared according to a published procedure. ${ }^{30} n$-BuLi (2.5 M solution in hexane), and other compounds were purchased from Sigma-Aldrich, FLuorochem and Acros Organics. Concentration of BuLi was determined by titration using menthol and 1,10-phenanthroline before use.

General procedure for the synthesis of starting phosphates 2a-2d (GP1). 1,3-Dicarbonyl compound was added to a suspension of sodium hydride (1.25 equiv.) in dry THF ( $5 \mathrm{~mL} / 1 \mathrm{mmol})$ cooled to $0{ }^{\circ} \mathrm{C}$. The resultant mixture was stirred for $30 \mathrm{~min}$ at $23{ }^{\circ} \mathrm{C}$ followed by addition of dialkyl chlorophosphate $(1.25$ equiv.). Then the reaction mixture was stirred for $2 \mathrm{~h}$ at $23{ }^{\circ} \mathrm{C}$. The crude reaction mixture was quenched with saturated aqueous solution of ammonium chloride $(1 \mathrm{~mL} / 1 \mathrm{mmol})$, the organic layer was separated and the water layer was extracted with ether $(3 \times 3 \mathrm{~mL} / 1 \mathrm{mmol})$. Combined organic layers were dried over $\mathrm{MgSO}_{4}$, the solvents were removed under reduce pressure and column chromatography (Silica gel) gave the product.

Ethyl (Z)-3-((diphenoxyphosphoryl)oxy)but-2-enoate

(2b). Prepared according to the GP1 from ethyl acetoacetate $(0.260 \mathrm{~g}$, $2.0 \mathrm{mmol})$, NaH (0.060 g, $2.50 \mathrm{mmol})$ and difenyl chlorophosphate $(0.67 \mathrm{~g}, 2.5 \mathrm{mmol})$. Column chromatography (hexane/AcOEt $\left.3: 1, R_{\mathrm{f}}=0.45\right)$ gave $0.67 \mathrm{~g}(93 \%)$ of the title compound as a yellowish oil. ${ }^{1} \mathrm{H}$ NMR $\left(300 \mathrm{MHz}, \mathrm{CDCl}_{3}\right) \delta 7.39-$ $7.16(\mathrm{~m}, 10 \mathrm{H}), 5.43(\mathrm{~s}, 1 \mathrm{H}), 4.11(\mathrm{q}, J=7.1 \mathrm{~Hz}, 2 \mathrm{H}), 2.18-2.14(\mathrm{~m}$, $3 \mathrm{H}), 1.25(\mathrm{t}, J=7.1 \mathrm{~Hz}, 3 \mathrm{H}) ;{ }^{13} \mathrm{C} \mathrm{NMR}\left(75 \mathrm{MHz} \mathrm{CDCl}_{3}\right) \delta 163.2$ $(\mathrm{d}, J=1.7 \mathrm{~Hz}), 156.6(\mathrm{~d}, J=7.2 \mathrm{~Hz}), 150.4(\mathrm{~d}, J=7.6 \mathrm{~Hz}), 129.7$, $125.5(\mathrm{~d}, J=1.2 \mathrm{~Hz}), 120.1(\mathrm{~d}, J=4.9 \mathrm{~Hz}), 106.7(\mathrm{~d}, J=8.6 \mathrm{~Hz})$, 60.0, 21.5 (d, $J=1.2 \mathrm{~Hz}$ ), 14.0; HRMS (ESI) $\mathrm{m} / \mathrm{z}$ : calcd for $\mathrm{C}_{18} \mathrm{H}_{20} \mathrm{O}_{6} \mathrm{P}[\mathrm{M}+\mathrm{H}]^{+}$363.0992; found 363.0992.

Phenyl (Z)-3-((diethoxyphosphoryl)oxy)but-2-enoate

(2c). Prepared according to the GP1 from phenyl acetoacetate $(1.780 \mathrm{~g}, 10.0 \mathrm{mmol})$, NaH $(0.480 \mathrm{~g}, 12.0 \mathrm{mmol})$ and diethyl chlorophosphate $(2.070 \mathrm{~g}, 12.0 \mathrm{mmol})$. Column chromatography (hexane/AcOEt $2: 1, R_{\mathrm{f}}=0.21$ ) gave $2.61 \mathrm{~g}(83 \%)$ of the title compound as a yellowish oil. ${ }^{1} \mathrm{H}$ NMR (300 $\left.\mathrm{MHz}, \mathrm{CDCl}_{3}\right)$ $\delta$ 7.41-7.38 (m, 2H), 7.26-7.23 (m, 1H), 7.14-7.12 (m, 2H), 5.57 (s, 1H), 4.31-4.25 (m, 4H), $2.30(\mathrm{~s}, 3 \mathrm{H}), 1.38-1.35(\mathrm{~m}, 6 \mathrm{H}) ;{ }^{13} \mathrm{C}$ NMR (75 MHz, $\left.\mathrm{CDCl}_{3}\right) \delta 161.7(\mathrm{~d}, J=1.7 \mathrm{~Hz}), 160.2(\mathrm{~d}, J=6.3$ $\mathrm{Hz}), 150.4,129.2$, 125.5, 121.6, 104.3 (d, $J=8.5 \mathrm{~Hz}), 64.9$ (d, $J=$ $6.5 \mathrm{~Hz}), 21.7$ (d, $J=1.2 \mathrm{~Hz}), 15.9$ (d, $J=7.1 \mathrm{~Hz}) ;{ }^{31} \mathrm{P}$ NMR $(202$ $\mathrm{MHz} \mathrm{CDCl}_{3}$ ) $\delta-8.38$ (s); HRMS (ESI) $m / z$ : calcd for $\mathrm{C}_{14} \mathrm{H}_{19} \mathrm{O}_{6} \mathrm{P}$ $[\mathrm{M}+\mathrm{Na}]^{+}$337.0812; found 337.0815.

Phenyl (Z)-3-[(diethoxyphosphoryl)oxy]hept-2-enoate (2d). Prepared according to the GP1 from phenyl 3-oxoheptanoate (3.524 g, $16.0 \mathrm{mmol})$, NaH (0.768 g, $19.20 \mathrm{mmol})$ and diethyl chlorophosphate $(2.899 \mathrm{~g}, 16.80 \mathrm{mmol})$. Column chromatography (hexane/AcOEt $\left.3: 1, R_{\mathrm{f}}=0.26\right)$ gave $4.048 \mathrm{~g}(71 \%)$ of the title compound as a yellowish oil. ${ }^{1} \mathrm{H}$ NMR (300 $\mathrm{MHz}, \mathrm{CDCl}_{3}$ ):

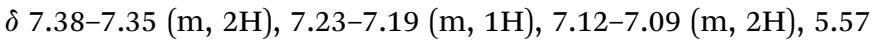
(s, 1H), 4.29-4.17 (m, 4H), 2.54-2.50 (m, 2H), 1.64-1.58 (m, 2H),
1.44-1.38 (m, 2H), $1.32(\mathrm{td}, J=7.1,1.2 \mathrm{~Hz}, 6 \mathrm{H}), 0.95(\mathrm{t}, J=$ $7.3 \mathrm{~Hz}, 3 \mathrm{H}) ;{ }^{13} \mathrm{C}$ NMR $\left(100 \mathrm{MHz}, \mathrm{CDCl}_{3}\right) \delta 164.1(\mathrm{~d}, J=6.9 \mathrm{~Hz})$, $162.0(\mathrm{~d}, J=1.9 \mathrm{~Hz}), 150.4,129.3,125.6,121.7,104.1(\mathrm{~d}, J=7.8$ $\mathrm{Hz}), 64.9$ (d, $J=6.4 \mathrm{~Hz}), 35.0(\mathrm{~d}, J=1.0 \mathrm{~Hz}), 28.4,22.0,16.0$ (d, $J$ $=7.3 \mathrm{~Hz}$ ), 13.7; HRMS (APCI) $m / z$ : calcd for $\mathrm{C}_{17} \mathrm{H}_{25} \mathrm{O}_{6} \mathrm{P}[\mathrm{M}+\mathrm{H}]^{+}$ 357.1462; found 357.1463.

General procedure for the synthesis of phosphordiamidates 2e-2k (GP2). 1,3-Dicarbonyl compound was added to a suspension of sodium hydride ( 1.25 equiv.) in dry THF ( $5 \mathrm{~mL} / 1 \mathrm{mmol})$ cooled to $0{ }^{\circ} \mathrm{C}$. The resultant mixture was stirred for $30 \mathrm{~min}$ at $23{ }^{\circ} \mathrm{C}$ followed by addition of bis( $N, N$-dimethylamino)phosphoryl chloride (1.0 equiv.). Then the reaction mixture was stirred for $75 \mathrm{~h}$ at $23{ }^{\circ} \mathrm{C}$. The crude reaction mixture was quenched with saturated aqueous solution of ammonium chloride $(1 \mathrm{~mL} / 1 \mathrm{mmol})$, the organic layer was separated and the water layer was extracted with AcOEt $(2 \times 3 \mathrm{~mL} / 1 \mathrm{mmol})$. Combined organic layers were washed with brine $(3 \mathrm{~mL} / 1$ mmol) dried over $\mathrm{MgSO}_{4}$, the solvents were removed under reduce pressure and column chromatography (Silica gel) gave the product.

Ethyl (Z)-3-[(bis(N,N-dimethylamino)phosphoryl)oxy]but-2-enoate (2e). Prepared according to the GP2 from ethyl acetoacetate (13.01 g, $100.0 \mathrm{mmol})$, NaH $(4.42 \mathrm{~g}, 110.0 \mathrm{mmol})$ and $\operatorname{bis}(N, N-$ dimethylamino)phosphoryl chloride (18.83 g, $110.0 \mathrm{mmol})$. Column chromatography (AcOEt, $R_{\mathrm{f}}=0.33$ ) gave $12.74 \mathrm{~g}(54 \%)$ of the title compound as a yellowish oil. ${ }^{1} \mathrm{H}$ NMR $\left(500 \mathrm{MHz}, \mathrm{CDCl}_{3}\right)$ $\delta 5.16(\mathrm{~s}, 1 \mathrm{H}), 4.07$ (q, $J=7.1 \mathrm{~Hz}, 2 \mathrm{H}), 2.69$ (d, $J=10.2 \mathrm{~Hz}, 12 \mathrm{H})$, $2.11(\mathrm{~s}, 3 \mathrm{H}), 1.20(\mathrm{dd}, J=7.8,6.5 \mathrm{~Hz}, 3 \mathrm{H}) ;{ }^{13} \mathrm{C}$ NMR $(126 \mathrm{MHz}$, $\left.\mathrm{CDCl}_{3}\right) \delta 164.0,159.7(\mathrm{~d}, J=5.8 \mathrm{~Hz}), 103.6(\mathrm{~d}, J=7.5 \mathrm{~Hz}), 59.4,36.4$, $22.1(\mathrm{~d}, J=2.1 \mathrm{~Hz}), 14.3 ;{ }^{31} \mathrm{P}$ NMR $\left(202 \mathrm{MHz}, \mathrm{CDCl}_{3}\right) \delta 15.02(\mathrm{~s})$; HRMS (ESI) $m / z$ : calcd for $\mathrm{C}_{10} \mathrm{H}_{21} \mathrm{~N}_{2} \mathrm{O}_{4} \mathrm{P}[\mathrm{M}+\mathrm{Na}]+287.1131$; found 287.1135 .

Ethyl (Z)-3-[(bis(N,N-dimethylamino)phosphoryl)oxy]hex-2enoate (2f). Prepared according to the GP2 from ethyl 3-oxohexanoate $(4.746 \mathrm{~g}, 30.0 \mathrm{mmol}), \mathrm{NaH}(1.441 \mathrm{~g}, 36.0 \mathrm{mmol})$ and bis( $N, N$-dimethylamino)phosphoryl chloride $(5.373 \mathrm{~g}, 31.50$ $\mathrm{mmol}$ ). Column chromatography (AcOEt, $R_{\mathrm{f}}=0.32$ ) gave $4.648 \mathrm{~g}$ (53\%) of the title compound as a yellowish oil. ${ }^{1} \mathrm{H}$ NMR (500 $\left.\mathrm{MHz} \mathrm{CDCl}_{3}\right) \delta 5.24-5.22(\mathrm{~m}, 1 \mathrm{H}), 4.12-4.07(\mathrm{~m}, 2 \mathrm{H}), 2.72(\mathrm{~d}, J=$ $10.2 \mathrm{~Hz}, 12 \mathrm{H}), 2.44-2.38$ (m, 2H), 1.61-1.54 (m, 2H), 1.26-1.21 $(\mathrm{m}, 3 \mathrm{H}), 0.97-0.92(\mathrm{~m}, 3 \mathrm{H}) ;{ }^{13} \mathrm{C} \mathrm{NMR}\left(100 \mathrm{MHz}, \mathrm{CDCl}_{3}\right) \delta 164.1$, 163.5 (d. $J=6.2 \mathrm{~Hz}), 103.1$ (d, $J=7.2 \mathrm{~Hz}), 59.5,37.3,36.6$ (d, $J=$ $4.3 \mathrm{~Hz}$ ), 20.0, 14.3, 13.5 (d, $J=1.4 \mathrm{~Hz}$ ); HRMS (APCI) $\mathrm{m} / \mathrm{z}$ : calcd for $\mathrm{C}_{12} \mathrm{H}_{25} \mathrm{~N}_{2} \mathrm{O}_{4} \mathrm{P}[\mathrm{M}+\mathrm{H}]^{+}$293.1625; found 293.1628.

Ethyl (Z)-3-((bis(N,N-dimethylamino)phosphoryl)oxy)-3phenylacrylate (2g). Prepared according to the GP2 from ethyl benzoylacetate $(3.840 \mathrm{~g}, 20.0 \mathrm{mmol}), \mathrm{NaH}(0.960 \mathrm{~g}, 24.0 \mathrm{mmol})$ and bis( $N, N$-dimethylamino)phosphoryl chloride $(4.120 \mathrm{~g}, 24.0$ $\mathrm{mmol}$ ). Column chromatography (AcOEt, $\left.R_{\mathrm{f}}=0.38\right)$ gave $3.86 \mathrm{~g}$ (55\%) of the title compound as a yellowish oil. ${ }^{1} \mathrm{H}$ NMR (500 $\left.\mathrm{MHz} \mathrm{CDCl}_{3}\right) \delta$ 7.64-7.52 (m, 2H), 7.43-7.42 (m, 3H), $5.81(\mathrm{~s}$, $1 \mathrm{H}), 4.21$ (q, $J=7.1 \mathrm{~Hz}, 2 \mathrm{H}), 2.61(\mathrm{~d}, J=10.1 \mathrm{~Hz}, 12 \mathrm{H}), 1.34(\mathrm{t}, J$ $=7.1 \mathrm{~Hz}, 3 \mathrm{H}) ;{ }^{13} \mathrm{C} \mathrm{NMR}\left(126 \mathrm{MHz}, \mathrm{CDCl}_{3}\right) \delta 164.3(\mathrm{~d}, J=2.1 \mathrm{~Hz})$, $158.6(\mathrm{~d}, J=6.7 \mathrm{~Hz}), 136.0(\mathrm{~d}, J=1.8 \mathrm{~Hz}), 130.2,128.3,127.4$, $107.1(\mathrm{~d}, J=6.3 \mathrm{~Hz}), 60.1,36.6(\mathrm{~d}, J=4.6 \mathrm{~Hz}), 14.3 ;{ }^{31} \mathrm{P}$ NMR $\left(202 \mathrm{MHz}, \mathrm{CDCl}_{3}\right) \delta 15.23(\mathrm{~s})$. HRMS (ESI) $\mathrm{m} / \mathrm{z}$ : calcd for $\mathrm{C}_{15} \mathrm{H}_{23} \mathrm{~N}_{2} \mathrm{O}_{4} \mathrm{P}[\mathrm{M}+\mathrm{Na}]^{+}$349.1288; found 349.1294. 
Ethyl 2-((bis( $N, N$-dimethylamino)phosphoryl)oxy)cyclohex-1ene-1-carboxylate (2h). Prepared according to the GP2 from ethyl 2-oxocyclohexane-1-carboxylate (3.404 g, $20 \mathrm{mmol}), \mathrm{NaH}$ (0.960 g, $24 \mathrm{mmol})$ and bis $(N, N$-dimethylamino)phosphoryl chloride (3.582 g; $21.0 \mathrm{mmol}$ ). Column chromatography (AcOEt, $\left.R_{\mathrm{f}}=0.21\right)$ gave $4.578 \mathrm{~g}(75 \%)$ of the title compound as a yellowish oil. ${ }^{1} \mathrm{H}$ NMR $\left(300 \mathrm{MHz}, \mathrm{CDCl}_{3}\right) \delta 4.16(\mathrm{qd}, J=7.2$, $1.2 \mathrm{~Hz}, 2 \mathrm{H}), 2.68$ (d, $J=10.1,12 \mathrm{H}), 2.48-2.44(\mathrm{~m}, 2 \mathrm{H}), 2.34-2.31$ (m, 2H), 1.71-1.56 (m, 4H), 1.28-1.24 (m, 3H); ${ }^{13} \mathrm{C}$ NMR (100 $\left.\mathrm{MHz} \mathrm{CDCl}_{3}\right) \delta 153.6(\mathrm{~d}, J=6.6 \mathrm{~Hz}), 113.9(\mathrm{~d}, J=7.4 \mathrm{~Hz}), 111.6$ $(\mathrm{d}, J=4.7 \mathrm{~Hz}), 60.0,36.5(\mathrm{~d}, J=4.3 \mathrm{~Hz}), 29.3(\mathrm{~d}, J=1.7 \mathrm{~Hz}), 25.6$, 22.3, 21.6, 14.2; ${ }^{31} \mathrm{P}$ NMR (202 $\mathrm{MHz}, \mathrm{CDCl}_{3}$ ): $\delta$ 14.88; HRMS (APCI) $m / z$ : calcd for $\mathrm{C}_{13} \mathrm{H}_{25} \mathrm{~N}_{2} \mathrm{O}_{4} \mathrm{P}[\mathrm{M}+\mathrm{H}]^{+} 305.1625$; found 305.1628 .

Methyl 2-[(bis(N,N-dimethylamino)phosphoryl)oxy]cyclopent-1ene-1-carboxylate (2i). Prepared according to the GP2 from ethyl 2-oxocyclopentane-1-carboxylate $(1.424 \mathrm{~g}, 10.0 \mathrm{mmol})$, $\mathrm{NaH}(0.480 \mathrm{~g}, 12.0 \mathrm{mmol})$ and bis( $N, N$-dimethylamino)phosphoryl chloride $(1.791 \mathrm{~g} ; 10.50 \mathrm{mmol})$. Column chromatography (AcOEt, $\left.R_{\mathrm{f}}=0.28\right)$ gave $2.127 \mathrm{~g}(77 \%)$ of the title compound as a yellowish oil. ${ }^{1} \mathrm{H}$ NMR $\left(400 \mathrm{MHz}, \mathrm{CDCl}_{3}\right) \delta 3.69(\mathrm{~s}, 3 \mathrm{H}), 2.72-$ $2.70(\mathrm{~m}, 14 \mathrm{H}), 2.57-2.57(\mathrm{~m}, 2 \mathrm{H}), 1.93-1.85(\mathrm{~m}, 2 \mathrm{H}) ;{ }^{13} \mathrm{C}$ NMR $\left(100 \mathrm{MHz}, \mathrm{CDCl}_{3}\right) \delta 164.6,159.6(\mathrm{~d}, J=5.3 \mathrm{~Hz}), 112.2(\mathrm{~d}, J=7.6$ $\mathrm{Hz}), 50.9,36.4$ (d, $J=4.4 \mathrm{~Hz}), 33.5(\mathrm{~d}, J=1.9 \mathrm{~Hz}), 28.7,19.3 ;{ }^{31} \mathrm{P}$ NMR (202 MHz, $\mathrm{CDCl}_{3}$ ): $\delta$ 15.63; HRMS (APCI) $\mathrm{m} / \mathrm{z}$ : calcd for $\mathrm{C}_{11} \mathrm{H}_{21} \mathrm{~N}_{2} \mathrm{O}_{4} \mathrm{P}[\mathrm{M}+\mathrm{H}]^{+}=277.1312$; found 277.1315.

Methyl 2-[(bis(N,N-dimethylamino)phosphoryl)oxy]cyclohept-1ene-1-carboxylate (2j). Prepared according to the GP2 from ethyl 2-oxocycloheptane-1-carboxylate $(2.558 \mathrm{~g}, 15.0 \mathrm{mmol})$, $\mathrm{NaH}(0.721 \mathrm{~g}, 18.0 \mathrm{mmol})$ and bis( $N, N$-dimethylamino $)$ phosphoryl chloride (2.729 g, $16.0 \mathrm{mmol})$. Column chromatography (AcOEt, $\left.R_{\mathrm{f}}=0.25\right)$ gave $3.142 \mathrm{~g}(69 \%)$ of the title compound as a colorless liquid. ${ }^{1} \mathrm{H}$ NMR $\left(300 \mathrm{MHz}, \mathrm{CDCl}_{3}\right) \delta 3.55(\mathrm{~s}, 3 \mathrm{H})$, 2.58-2.43 (m, 14H), 2.26-2.26 (m, 2H), 1.59-1.37 (m, 6H); ${ }^{13} \mathrm{C}$ NMR (100 MHz, $\left.\mathrm{CDCl}_{3}\right) \delta 167.6(\mathrm{~d}, J=1.7 \mathrm{~Hz}), 158.2(\mathrm{~d}, J=7.3$ $\mathrm{Hz}), 118.7$ (d, $J=7.4 \mathrm{~Hz}), 51.1,36.1$ (d, $J=4.2 \mathrm{~Hz}), 33.6$ (d, $J=$ $1.6 \mathrm{~Hz}), 31.2,27.4,25.8(\mathrm{~d}, J=1.4 \mathrm{~Hz}), 23.9(\mathrm{~d}, J=0.6 \mathrm{~Hz}) ;{ }^{31} \mathrm{P}$ NMR (202 MHz, $\mathrm{CDCl}_{3}$ ): $\delta$ 14.67; HRMS (APCI) $\mathrm{m} / \mathrm{z}$ : calcd for $\mathrm{C}_{13} \mathrm{H}_{25} \mathrm{~N}_{2} \mathrm{O}_{4} \mathrm{P}[\mathrm{M}+\mathrm{H}]^{+}$305.1625; found 305.1626.

Ethyl (Z)-3-[(bis(N,N-dimethylamino)phosphoryl)oxy]-2methylbut-2-enoate (2k). Prepared according to the GP2 from ethyl 2-methylacetoacetate ( $2.88 \mathrm{~g}, 20 \mathrm{mmol})$, NaH $(0.960 \mathrm{~g}, 24.0$ mmol) and bis( $N, N$-dimethylamino)phosphoryl chloride $(4.120 \mathrm{~g}, 24.0 \mathrm{mmol})$. Column chromatography (AcOEt, $R_{\mathrm{f}}=$ $0.33)$ gave $2.60 \mathrm{~g}(50 \%)$ of the title compound as a yellowish oil. ${ }^{1} \mathrm{H}$ NMR $\left(500 \mathrm{MHz}, \mathrm{CDCl}_{3}\right) \delta 4.17(\mathrm{q}, J=7.1 \mathrm{~Hz}, 2 \mathrm{H}), 2.67(\mathrm{~d}, J=$ $10.1 \mathrm{~Hz}, 12 \mathrm{H}), 2.11(\mathrm{~s}, 3 \mathrm{H}), 1.83(\mathrm{~s}, 3 \mathrm{H}), 1.27$ (t, $J=7.1 \mathrm{~Hz}, 3 \mathrm{H})$; ${ }^{13} \mathrm{C}$ NMR $\left(126 \mathrm{MHz}, \mathrm{CDCl}_{3}\right) \delta 167.3,150.0(\mathrm{~d}, J=6.9 \mathrm{~Hz}), 112.5$ $(\mathrm{d}, J=7.8 \mathrm{~Hz}), 60.2,36.5(\mathrm{~d}, J=3.8 \mathrm{~Hz}), 18.2(\mathrm{~d}, J=1.6 \mathrm{~Hz}), 14.7$, 14.2; ${ }^{31} \mathrm{P}$ NMR (202 MHz, $\mathrm{CDCl}_{3}$ ) $\delta 15.08$ (s); HRMS (ESI) $\mathrm{m} / z$ : calcd for $\mathrm{C}_{11} \mathrm{H}_{23} \mathrm{~N}_{2} \mathrm{O}_{4} \mathrm{P}[\mathrm{M}+\mathrm{Na}]^{+}$301.1288; found 301.1289.

General procedure for the synthesis of substituted buta-1,3dienes 3 (GP3). $n$-Butyllithium (4.0 equiv.) was added to a solution of aryl halide (4.1 equiv.) in dry THF ( $3 \mathrm{~mL} / 1 \mathrm{mmol}$ ) cooled to $-78{ }^{\circ} \mathrm{C}$ and the resultant mixture was stirred for $30 \mathrm{~min}$ at $-78{ }^{\circ} \mathrm{C}$ followed by addition of enol phosphates $2 \mathbf{a}-\mathbf{2 d}(1$ equiv.). Then the crude reaction mixture was stirred for $2 \mathrm{~h}$ at $23{ }^{\circ} \mathrm{C}$. The reaction mixture was quenched by phosphoric acid ( 1 $\mathrm{mL} / 1 \mathrm{mmol}, 85 \mathrm{wt} \%$ ) and the reaction mixture was stirred for $1 \mathrm{~h}$ at $60{ }^{\circ} \mathrm{C}$. Then the reaction mixture was cooled to $23{ }^{\circ} \mathrm{C}$, extracted with ether $\left(2 \times 4 \mathrm{~mL} \mathrm{mmol}^{-1}\right)$. The combined organic phases were washed with water $\left(1 \times 4 \mathrm{~mL} \mathrm{mmol}^{-1}\right)$, aqueous solution of saturated sodium bicarbonate $\left(1 \times 4 \mathrm{~mL} \mathrm{mmol}^{-1}\right)$ and brine $\left(1 \times 4 \mathrm{~mL} \mathrm{mmol}^{-1}\right)$. The organic layer was dried over $\mathrm{MgSO}_{4}$, the solvents were removed under reduce pressure and the product was obtained by column chromatography (Silica gel).

1,1,3-Triphenylbuta-1,3-diene (3a). Prepared according to the GP3 from bromobenzene $(0.320 \mathrm{~g}, 2.05 \mathrm{mmol}),{ }^{n} \mathrm{BuLi}(0.83 \mathrm{~mL}$, $2.0 \mathrm{mmol}, 2.4 \mathrm{M}$ ) and enol phosphate $2 \mathrm{c}(0.160 \mathrm{~g}, 0.5 \mathrm{mmol})$. Column chromatography (hexane/toluene $99: 1, R_{\mathrm{f}}=0.47$ ) gave $0.092 \mathrm{~g}(65 \%)$ of the title compound as a colorless oil. ${ }^{1} \mathrm{H}$ NMR (300 MHz, $\left.\mathrm{CDCl}_{3}\right) \delta 7.40-7.15(\mathrm{~m}, 15 \mathrm{H}), 6.74(\mathrm{~d}, J=1.2 \mathrm{~Hz}, 1 \mathrm{H})$, $5.39(\mathrm{~d}, J=1.4 \mathrm{~Hz}, 1 \mathrm{H}), 5.03(\mathrm{t}, J=1.4 \mathrm{~Hz}, 1 \mathrm{H})$, in accordance with literature. ${ }^{22}$

1,1,3-Tris(4-methylphenyl)buta-1,3-diene (3b). Prepared according to the GP3 from 4-bromotoluene $(0.350 \mathrm{~g}, 2.05$ $\mathrm{mmol}),{ }^{n} \mathrm{BuLi}(0.83 \mathrm{~mL}, 2.0 \mathrm{mmol}, 2.4 \mathrm{M}$ ) and enol phosphate 2c $(0.160 \mathrm{~g}, 0.5 \mathrm{mmol})$. Column chromatography (hexane, $R_{\mathrm{f}}=$ $0.34)$ gave $0.089 \mathrm{~g}(55 \%)$ of the title compound as a colorless oil. ${ }^{1} \mathrm{H}$ NMR (400 MHz, $\mathrm{CDCl}_{3}$ ) $\delta$ 7.37-7.37 (m, 2H), 7.26-7.24 (m, 2H), 7.15-7.08 (m, 6H), 7.05-7.03 (m, 2H), 6.67-6.66 (m, 1H), 5.38-5.37 (m, 1H), 4.97-4.96 (m, 1H), $2.38(\mathrm{~s}, 3 \mathrm{H}), 2.35(\mathrm{~s}, 3 \mathrm{H})$, $2.33(\mathrm{~s}, 3 \mathrm{H}) ;{ }^{13} \mathrm{C} \mathrm{NMR}\left(101 \mathrm{MHz}, \mathrm{CDCl}_{3}\right) \delta 145.0,144.4,140.6$, 138.1, 137.4, 137.3, 137.2, 136.6, 130.0, 128.9, 128.8, 128.7, 127.9, 127.5, 126.5, 115.9, 21.3, 21.2, 21.1; HRMS (APCI) $m / z$ : calcd for $\mathrm{C}_{25} \mathrm{H}_{24}[\mathrm{M}+\mathrm{H}]^{+} 325.1951$ found 325.1945.

1,1,3-Tris(4-methoxyphenyl)buta-1,3-diene (3c). Prepared according to the GP3 from 4-bromoanisole ( $0.38 \mathrm{~g}, 2.05 \mathrm{mmol})$, ${ }^{n} \mathrm{BuLi}(0.83 \mathrm{~mL}, 2.0 \mathrm{mmol}, 2.4 \mathrm{M})$ and enol phosphate 2c (0.160 g, $0.5 \mathrm{mmol})$. Column chromatography (hexane/AcOEt $\left.20: 1, R_{\mathrm{f}}=0.23\right)$ gave $0.104 \mathrm{~g}(56 \%)$ of the title compound as a yellowish oil. ${ }^{1} \mathrm{H}$ NMR $\left(400 \mathrm{MHz}, \mathrm{CDCl}_{3}\right) \delta 7.36-7.33(\mathrm{~m}, 2 \mathrm{H})$, 7.29-7.26 (m, 2H), 7.09-7.06 (m, 2H), 6.87-6.84 (m, 2H), 6.80$6.72(\mathrm{~m}, 4 \mathrm{H}), 6.59-6.57(\mathrm{~m}, 1 \mathrm{H}), 5.31-5.31(\mathrm{~m}, 1 \mathrm{H}), 4.95-4.93$ (m, 1H), $3.83(\mathrm{~s}, 3 \mathrm{H}), 3.79(\mathrm{~s}, 3 \mathrm{H}), 3.77(\mathrm{~s}, 3 \mathrm{H}) ;{ }^{13} \mathrm{C}$ NMR (101 $\left.\mathrm{MHz}, \mathrm{CDCl}_{3}\right) \delta 159.2,159.1,158.6,144.9,143.6,136.1,133.5$, 132.7, 131.3, 129.2, 127.8, 126.6, 115.1, 113.5, 113.4, 113.3, 55.3, 55.3, 55.2. HRMS (APCI) $m / z$ : calcd for $\mathrm{C}_{25} \mathrm{H}_{24} \mathrm{O}_{3}[\mathrm{M}+\mathrm{H}]^{+}$ 373.1798; found 373.1793 .

1,1,3-Tris[(1,1'-biphenyl)-4-yl]buta-1,3-diene (3d). Prepared according to the GP3 from 4-bromobiphenyl (0.48 g, 2.05 $\mathrm{mmol}),{ }^{n} \mathrm{BuLi}(0.83 \mathrm{~mL}, 2.0 \mathrm{mmol}, 2.4 \mathrm{M}$ ) and enol phosphate $2 \mathrm{c}$ $(0.160 \mathrm{~g}, 0.5 \mathrm{mmol})$. Column chromatography (hexane/AcOEt $\left.20: 1, R_{\mathrm{f}}=0.57\right)$ gave $0.092 \mathrm{~g}(64 \%)$ of the title compound as a white solid, $\mathrm{mp}=64.0-66.0{ }^{\circ} \mathrm{C} .{ }^{1} \mathrm{H} \mathrm{NMR}\left(400 \mathrm{MHz}, \mathrm{CDCl}_{3}\right)$ $\delta$ 7.65-7.25 (m, 17H), 6.89 (s 1H), $5.51(\mathrm{~s}, 1 \mathrm{H}), 5.20(\mathrm{~s}, 1 \mathrm{H}) ;{ }^{13} \mathrm{C}$ NMR (101 MHz, $\left.\mathrm{CDCl}_{3}\right) \delta 145.1,143.9,142.0,140.82,140.80$, $140.7,140.5$, 140.3, 139.9, 139.6, 138.9, 130.7, 128.8, 128.71, $128.69,128.53,128.48,127.4,127.29,127.22,127.20,127.1$, 127.04, 127.02, 126.95, 126.8, 126.6, 118.0; HRMS (APCI) $m / z$ : calcd for $\mathrm{C}_{40} \mathrm{H}_{30}[\mathrm{M}+\mathrm{H}]^{+}$511.2420; found 511.2397. 
1,1,3-Tris(2-methoxyphenyl)buta-1,3-diene (3e). Prepared according to the GP3 from 2-bromoanisole $(0.380 \mathrm{~g}, 2.05 \mathrm{mmol})$, ${ }^{n} \mathrm{BuLi}(0.83 \mathrm{~mL}, 2.0 \mathrm{mmol}, 2.4 \mathrm{M})$ and enol phosphate $2 \mathrm{c}$ (0.160 g, $0.5 \mathrm{mmol}$ ). Column chromatography (hexane/AcOEt $\left.20: 1, R_{\mathrm{f}}=0.22\right)$ gave $0.132 \mathrm{~g}(71 \%)$ of the title compound as a yellowish oil. ${ }^{1} \mathrm{H}$ NMR $\left(400 \mathrm{MHz}, \mathrm{CDCl}_{3}\right) \delta 7.30-7.19(\mathrm{~m}, 2 \mathrm{H})$, 7.12-7.10 (m, 1H), 7.03-6.87 (m, 4H), 6.84-6.81 (m, 2H), 6.76$6.72(\mathrm{~m}, 1 \mathrm{H}), 6.70-6.66(\mathrm{~m}, 1 \mathrm{H}), 6.51-6.45(\mathrm{~m}, 2 \mathrm{H}), 5.36-5.35$ $(\mathrm{m}, 1 \mathrm{H}), 5.21-5.20(\mathrm{~m}, 1 \mathrm{H}), 3.71(\mathrm{~s}, 3 \mathrm{H}), 3.61(\mathrm{~s}, 6 \mathrm{H}) ;{ }^{13} \mathrm{C} \mathrm{NMR}$ $\left(101 \mathrm{MHz}, \mathrm{CDCl}_{3}\right) \delta 157.3,156.3,156.0,144.8,136.0,133.8$, $132.2,130.8,130.4,130.4,130.1,129.8,128.1,128.0,127.7$, 120.4, 120.3, 119.5, 119.1, 111.8, 109.5, 109.4, 55.8, 55.0, 54.9; HRMS (APCI) $m / z$ : calcd for $\mathrm{C}_{25} \mathrm{H}_{24} \mathrm{O}_{3}[\mathrm{M}+\mathrm{H}]^{+} 373.1798$; found 373.1789 .

(E/Z)-1,1,3-Triphenylhepta-1,3-diene (3f). Prepared according to the GP3 from bromobenzene $(0.320 \mathrm{~g}, 2.05 \mathrm{mmol}),{ }^{n} \mathrm{BuLi}$ (0.83 mL, $2.0 \mathrm{mmol}, 2.4 \mathrm{M})$ and enol phosphate $2 \mathrm{~d}(0.178 \mathrm{~g}, 0.5$ mmol). Column chromatography (hexane, $R_{\mathrm{f}}=0.35$ ) gave $0.101 \mathrm{~g}(62 \%, E / Z=79: 21)$ of the title compound as a yellowish oil. ${ }^{1} \mathrm{H}$ NMR (300 MHz, $\left.\mathrm{CDCl}_{3}\right) \delta 7.37-7.10(\mathrm{~m}, 15 \mathrm{H}, E$ and $Z$ ), 6.69-6.68 (m, 1H, $E$ and $Z$ ), 5.77-5.73 (m, 1H, $\left.E^{\text {major }}\right), 5.64-5.61$ (m, $\left.1 \mathrm{H}, Z^{\text {minor }}\right), 1.99-1.95(\mathrm{~m}, 2 \mathrm{H}, E$ and $Z), 1.24-1.15(\mathrm{~m}, 2 \mathrm{H}, E$ and $Z$ ), $0.84\left(\mathrm{t}, J=6.0 \mathrm{~Hz}, 3 \mathrm{H}, E^{\text {major }}\right), 0.72(\mathrm{t}, J=6.0 \mathrm{~Hz}, 3 \mathrm{H}$, $\left.Z^{\text {minor }}\right) ;{ }^{13} \mathrm{C} \mathrm{NMR}\left(100 \mathrm{MHz}, \mathrm{CDCl}_{3}\right) \delta 145.1,143.6^{\text {minor }}$, $143.3^{\text {major }}, \quad 141.8^{\text {major }}, \quad 141.6^{\text {minor }}, \quad 140.4^{\text {minor }}, 140.3^{\text {major }}$, $139.7^{\text {minor }}, \quad 138.2^{\text {minor }}, \quad 137.3^{\text {major }}, \quad 135.6^{\text {major }}, \quad 131.6^{\text {major }}$, $131.3^{\text {minor }}, 130.4,129.9,129.2$, 128.2, 128.1, 128.0, 127.9, 127.7, 127.6, 127.5, 127.1, 127.0, 126.7, 126.50, 126.47, 126.4, 126.3, $31.9^{\text {major }}, 31.2^{\text {minor }}, 22.7^{\text {minor }}, 22.2^{\text {major }}, 14.0^{\text {major }}, 13.7^{\text {minor }}$; HRMS (APCI) $m / z$ : calcd for $\mathrm{C}_{25} \mathrm{H}_{24}[\mathrm{M}+\mathrm{H}]^{+} 325.1951$; found 325.1953 .

1,1,3-Tris(4-methylphenyl)hepta-1,3-diene (3g). Prepared according to the GP3 from 4-bromotoluene $(0.212 \mathrm{~g}, 1.23$ $\mathrm{mmol}$ ), ${ }^{n}$ BuLi (0.49 mL, 2.40 M, $1.18 \mathrm{mmol}$ ), and enol phosphate 2d $(0.107 \mathrm{~g}, 0.30 \mathrm{mmol})$. Column chromatography (hexane, $\left.R_{\mathrm{f}}=0.28\right)$ gave the title compound $0.056 \mathrm{~g}(51 \%, E / Z=$ $83: 17)$ as a yellowish oil. ${ }^{1} \mathrm{H}$ NMR $\left(300 \mathrm{MHz}, \mathrm{CDCl}_{3}\right) \delta 7.31-7.24$ (m, 3H), 7.14-6.95 (m, 9H), 6.56-6.55 (m, $\left.1 \mathrm{H}, Z^{\text {minor }}\right), 6.53-6.52$ $\left(\mathrm{s}, 1 \mathrm{H}, E^{\text {major }}\right), 5.68\left(\mathrm{t}, J=6.0 \mathrm{~Hz}, 1 \mathrm{H}, E^{\text {major }}\right), 5.53(\mathrm{t}, J=6.0 \mathrm{~Hz}$, $\left.1 \mathrm{H}, Z^{\text {minor }}\right), 2.37\left(\mathrm{~s}, 3 \mathrm{H}, E^{\text {major }}\right), 2.32\left(\mathrm{~s}, 3 \mathrm{H}, Z^{\text {minor }}\right), 2.30(\mathrm{~s}, 3 \mathrm{H}$, $\left.E^{\text {major }}\right), 2.29\left(\mathrm{~s}, 3 \mathrm{H}, Z^{\text {minor }}\right), 2.27\left(\mathrm{~s}, 3 \mathrm{H}, E^{\text {major }}\right), 1.96-1.84(\mathrm{~m}, 2 \mathrm{H}$, $E$ and $Z), 1.19-1.09(\mathrm{~m}, 2 \mathrm{H}, E$ and $Z), 0.80(\mathrm{t}, J=6.0 \mathrm{~Hz}, 3 \mathrm{H}$, $\left.E^{\text {major }}\right), 0.69\left(\mathrm{t}, J=6.0 \mathrm{~Hz}, 3 \mathrm{H}, Z^{\text {minor }}\right) ;{ }^{13} \mathrm{C} \mathrm{NMR}$ (75 MHz, $\mathrm{CDCl}_{3}$, only major peaks are reported) $\delta 144.7,140.7,138.9,137.5$, 137.2 , 136.9, 136.5, 136.1, 130.4, 130.2, 129.7, 129.6, 129.0, 128.9, 128.8, 128.7, 128.7, 128.3, 128.1, 127.5, 126.4, 125.4, 31.9, 22.2, 21.2, 21.1, 21.0, 14.0; HRMS (APCI) $m / z$ : calcd for $\mathrm{C}_{28} \mathrm{H}_{30}$ $[\mathrm{M}+\mathrm{H}]^{+}$367.2420; found 367.2419.

1,1,3-Tris(4-methoxyphenyl)hepta-1,3-diene (3h). Prepared according to the GP3 from 4-bromoanisole ( $0.230 \mathrm{~g}, 1.23 \mathrm{mmol}$ ) a ${ }^{n} \mathrm{BuLi}(0.49 \mathrm{~mL}, 2.40 \mathrm{M}, 1.17 \mathrm{mmol})$ and enol phosphate 2d $(0.107 \mathrm{~g}, 0.30 \mathrm{mmol})$. Column chromatography (hexane/EtOAc $\left.20: 1, R_{\mathrm{f}}=0.35\right)$ gave $0.063 \mathrm{~g}(51 \%, E / Z=82: 18)$ of the title compound as a yellowish oil. ${ }^{1} \mathrm{H}$ NMR $\left(300 \mathrm{MHz} \mathrm{CDCl}_{3}\right) \delta 7.31-$ $7.26(\mathrm{~m}, 4 \mathrm{H}), 7.04-6.65(\mathrm{~m}, 8 \mathrm{H}), 6.54-6.53\left(\mathrm{~m}, 1 \mathrm{H}, Z^{\mathrm{minor}}\right), 6.48-$ $6.47\left(\mathrm{~m}, 1 \mathrm{H}, E^{\text {major }}\right), 5.63\left(\mathrm{t}, J=6.0 \mathrm{~Hz}, 1 \mathrm{H}, E^{\text {major }}\right), 5.58(\mathrm{t}, J=$ $\left.6.0 \mathrm{~Hz}, 1 \mathrm{H}, Z^{\text {minor }}\right), 3.83\left(\mathrm{~s}, 3 \mathrm{H}, E^{\text {major }}\right), 3.79\left(\mathrm{~s}, 3 \mathrm{H}, Z^{\text {minor }}\right), 3.77$ $\left(\mathrm{s}, 3 \mathrm{H}, E^{\text {major }}\right), 3.76\left(\mathrm{~s}, 3 \mathrm{H}, Z^{\text {minor }}\right), 3.75\left(\mathrm{~s}, 3 \mathrm{H}, E^{\text {major }}\right), 1.98-1.89$ (m, 2H, $E$ and $Z$ ), 1.26-1.15 (m, 2H, $E$ and $Z), 0.83(\mathrm{t}, J=6.0 \mathrm{~Hz}$, $\left.3 \mathrm{H}, E^{\text {major }}\right), 0.74\left(\mathrm{t}, J=6.0 \mathrm{~Hz}, 3 \mathrm{H}, Z^{\text {minor }}\right) ;{ }^{13} \mathrm{C} \mathrm{NMR}(100 \mathrm{MHz}$, $\left.\mathrm{CDCl}_{3}\right) \delta 159.1,158.5,158.4,143.9,136.8,136.2,134.6,133.0$, 131.0, 129.3, 129.2, 127.6, 124.4, 113.4, 113.3, 113.1, 113.0, 55.3, 55.2, 55.1, 31.8 $8^{\text {major }}, 31.3^{\text {minor }}, 22.9^{\text {minor }}, 22.3^{\text {major }}, 14.0^{\text {major }}$, $13.8^{\text {minor }}$; HRMS (APCI) $m / z$ : calcd for $\mathrm{C}_{28} \mathrm{H}_{30} \mathrm{O}_{3}[\mathrm{M}+\mathrm{H}]^{+}$ 415.2268; found 415.2264.

1,1,3-Tris[(1,1'-biphenyl)-4-yl]hepta-1,3-diene (3i). Prepared according to the GP3 from 4-bromobiphenyl (0.287 g, 1.23 $\mathrm{mmol}),{ }^{n} \mathrm{BuLi}(0.49 \mathrm{~mL}, 2.40 \mathrm{M}, 1.17 \mathrm{mmol})$ and enol phosphate 2d $(0.107 \mathrm{~g}, 0.30 \mathrm{mmol})$. Column chromatography (hexane $\rightarrow$ hexane/EtOAc $\left.30: 1, R_{\mathrm{f}}=0.1\right)$ gave $0.119 \mathrm{~g}(72 \%, E / Z=86: 14)$ of the title compound as a yellowish solid, $\mathrm{mp}=49.0-50.0{ }^{\circ} \mathrm{C}$. ${ }^{1} \mathrm{H}$ NMR $\left(300 \mathrm{MHz}, \mathrm{CDCl}_{3}\right.$ ) $\delta$ 7.65-7.06 (m, 27H, $E$ and $Z$ ), 6.85 (s, $\left.1 \mathrm{H}, Z^{\text {minor }}\right), 6.82\left(\mathrm{~s}, 1 \mathrm{H}, E^{\text {major }}\right), 5.86-5.78(\mathrm{~m}, 1 \mathrm{H}, E$ and $Z), 2.10-$ $2.01(\mathrm{~m}, 2 \mathrm{H}, E$ and $Z), 1.30-1.23(\mathrm{~m}, 2 \mathrm{H}, E$ and $Z), 0.88(\mathrm{t}, J=$ $\left.7.3 \mathrm{~Hz}, 3 \mathrm{H}, E^{\text {major }}\right), 0.76\left(\mathrm{~s}, J=7.3 \mathrm{~Hz}, 3 \mathrm{H}, Z^{\text {minor }}\right) ;{ }^{13} \mathrm{C} \mathrm{NMR}(75$ $\left.\mathrm{MHz}, \mathrm{CDCl}_{3}\right) \delta 144.3,142.4,142.0,141.0,140.9,140.8,140.8$, $140.7,140.4,139.8,139.3,139.3,139.2,139.0,138.5,138.4$, $137.0,136.6,132.0,131.6,130.8,130.4,129.8,128.8,128.7$, $128.6,128.6$, 128.0, 127.3, 127.2, 127.1, 127.0, 126.9, 126.9, $126.8,126.7,126.4,126.2,32.0^{\text {major }}, 31.4^{\text {minor }}, 22.8^{\text {minor }}$, $22.3^{\text {major }}, 14.1^{\text {major }}, 13.8^{\text {minor }}$; HRMS (APCI) $\mathrm{m} / \mathrm{z}$ : calcd for $\mathrm{C}_{43} \mathrm{H}_{36}[\mathrm{M}+\mathrm{H}]^{+}$553.2890; found 553.2894.

1,1,3-Tris(2-methoxyphenyl)hepta-1,3-diene (3j). Prepared according to the GP3 from 2-bromoanisole (0.230 g, $1.23 \mathrm{mmol}$ ), ${ }^{n} \mathrm{BuLi}(0.49 \mathrm{~mL}, 2.40 \mathrm{M}, 1.17 \mathrm{mmol})$ and enol phosphate 2d (0.107 g, $0.30 \mathrm{mmol}$ ). Column chromatography (hexane/EtOAc $\left.50: 1, R_{\mathrm{f}}=0.14\right)$ gave $0.090 \mathrm{~g}(72 \%, E / Z=71: 29)$ of the title compound as a yellowish oil. ${ }^{1} \mathrm{H}$ NMR $\left(300 \mathrm{MHz}, \mathrm{CDCl}_{3}\right) \delta 7.27-$ $7.12(\mathrm{~m}, 3 \mathrm{H}, E$ and $Z), 6.96-6.36(\mathrm{~m}, 10 \mathrm{H}, E$ and $Z), 5.80(\mathrm{t}, J=$ $\left.6.0 \mathrm{~Hz}, 1 \mathrm{H}, Z^{\text {minor }}\right), 5.49(\mathrm{t}, J=6.0 \mathrm{~Hz}, 1 \mathrm{H}), 3.71\left(\mathrm{~s}, 3 \mathrm{H}, E^{\text {major }}\right)$, $3.68\left(\mathrm{~s}, 3 \mathrm{H}, Z^{\text {minor }}\right), 3.62\left(\mathrm{~s}, 3 \mathrm{H}, Z^{\text {minor }}\right), 3.60\left(\mathrm{~s}, 3 \mathrm{H}, E^{\text {major }}\right), 3.59$ $\left(\mathrm{s}, 3 \mathrm{H}, Z^{\text {minor }}\right), 3.44\left(\mathrm{~s}, 3 \mathrm{H}, E^{\text {major }}\right), 2.24\left(\mathrm{q}, J=7.4 \mathrm{~Hz}, 2 \mathrm{H}, E^{\text {major }}\right)$, 1.78 (q, $\left.J=7.4 \mathrm{~Hz}, 2 \mathrm{H}, Z^{\text {minor }}\right), 1.46-1.40\left(\mathrm{~m}, 2 \mathrm{H}, E^{\text {major }}\right), 1.25-$ $1.22\left(\mathrm{~m}, 2 \mathrm{H}, Z^{\text {minor }}\right), 0.95\left(\mathrm{t}, J=7.3 \mathrm{~Hz}, 3 \mathrm{H}, E^{\text {major }}\right), 0.95(\mathrm{t}, J=$ $\left.7.3 \mathrm{~Hz}, 3 \mathrm{H}, Z^{\text {minor }}\right) ;{ }^{13} \mathrm{C}$ NMR $\left(100 \mathrm{MHz}, \mathrm{CDCl}_{3}\right) \delta 157.4,156.3$, $156.1,136.4,136.1,134.8,134.0,133.9,132.3,130.9,130.8$, $130.3,129.7,127.7,127.4,127.0,120.3,119.5,119.0,112.1$, 109.6, 109.4, 55.9, 55.1, 54.7, 31.3 ${ }^{\text {minor }}, 31.1^{\text {major }}, 22.7^{\text {major }}$, $22.6^{\text {minor }}, 14.0^{\text {major }}$, $13.9^{\text {minor }}$; HRMS (APCI) $\mathrm{m} / \mathrm{z}$ : calcd for $\mathrm{C}_{28} \mathrm{H}_{30} \mathrm{O}_{3}[\mathrm{M}+\mathrm{H}]^{+}$415.2268; found 415.2277.

1,1,3-Tris(2-thienyl)hepta-1,3-diene (3k). Prepared according to the GP3 from thiophene $(0.108 \mathrm{~g}, 1.28 \mathrm{mmol}),{ }^{n} \mathrm{BuLi}(0.51 \mathrm{~mL}$, $2.40 \mathrm{M}, 1.22 \mathrm{mmol})$, dry THF (3 $\mathrm{mL})$. The reaction mixture was stirred for $30 \mathrm{~min}$ at $0{ }^{\circ} \mathrm{C}$ followed by addition of enol phosphate 2d $(0.110 \mathrm{~g}, 0.31 \mathrm{mmol})$. Column chromatography (hexane, $R_{\mathrm{f}}=$ $0.14)$ gave $0.048 \mathrm{~g}(45 \%, E / Z=93: 7)$ of the title compound as a greenish oil. ${ }^{1} \mathrm{H}$ NMR $\left(300 \mathrm{MHz}, \mathrm{CDCl}_{3}\right) \delta 7.28-7.26(\mathrm{~m}, 1 \mathrm{H})$, 7.23-7.21 (m, 1H), 7.13-7.12 (m, 1H), 7.09-7.02 (m, 3H), 6.97$6.87(\mathrm{~m}, 3 \mathrm{H}), 6.69\left(\mathrm{~d}, J=1.2 \mathrm{~Hz}, 1 \mathrm{H}, E^{\text {major }}\right), 5.92(\mathrm{td}, J=7.4$, $\left.1.3 \mathrm{~Hz}, 1 \mathrm{H}, E^{\text {major }}\right), 5.92\left(\mathrm{td}, J=7.4,1.3 \mathrm{~Hz}, 1 \mathrm{H}, Z^{\text {minor }}\right), 2.24-$ $2.18\left(\mathrm{~m}, 2 \mathrm{H}, Z^{\text {minor }}\right), 2.07-2.01\left(\mathrm{~m}, 2 \mathrm{H}, E^{\text {major }}\right), 1.33-1.23(\mathrm{~m}$, $2 \mathrm{H}), 0.87\left(\mathrm{t}, J=7.4 \mathrm{~Hz}, 3 \mathrm{H}, E^{\text {major }}\right), 0.79(\mathrm{t}, J=7.4 \mathrm{~Hz}, 3 \mathrm{H}$, $\left.Z^{\text {minor }}\right) ;{ }^{13} \mathrm{C} \mathrm{NMR}\left(100 \mathrm{MHz}, \mathrm{CDCl}_{3}\right) \delta 146.9^{\text {minor }}, 145.9,145.3$, 
141.2 minor $^{\text {140. }}$ minor $, 140.1,136.2$, 131.3, 130.6, 128.5, 127.3, $127.1,126.5,126.4,126.2,125.1,125.1,123.7,123.3,31.7^{\text {major }}$, $31.6^{\text {minor }}, 22.6^{\text {minor }}, 22.2^{\text {major }}, 14.0^{\text {major }}, 13.8^{\text {minor }}$; HRMS (APCI) $m / z$ : calcd for $\mathrm{C}_{19} \mathrm{H}_{18} \mathrm{~S}_{3}[\mathrm{M}+\mathrm{H}]^{+}$343.0643; found 343.0649.

General procedure for conversion of phosphorodiamidates $2 \mathrm{e}-2 \mathrm{k}$ to $\boldsymbol{\alpha}, \boldsymbol{\beta}$-ketones $\mathbf{4 , 5}$ (GP4). Organolithium or Grignard reagent (2.5 equiv.) was added to a solution of phosphordiamidate $2 \mathbf{e}-2 \mathbf{k}$ in dry THF $(10 \mathrm{~mL} / 1 \mathrm{mmol})$ cooled to $-78{ }^{\circ} \mathrm{C}$. The resultant mixture was warmed to $23{ }^{\circ} \mathrm{C}$ and stirred for $2 \mathrm{~h}$ at $23{ }^{\circ} \mathrm{C}$. Then the reaction mixture was quenched by phosphoric acid ( $1 \mathrm{~mL} / 1 \mathrm{mmol}, 85 \mathrm{wt} \%)$ and the mixture was stirred for $1 \mathrm{~h}$ at $60{ }^{\circ} \mathrm{C}$. Then the reaction mixture was cooled to $23{ }^{\circ} \mathrm{C}$ and the mixture was extracted with ether $\left(2 \times 4 \mathrm{~mL} \mathrm{mmol}^{-1}\right)$. The combined organic phases were washed with water $(1 \times 4 \mathrm{~mL}$ $\left.\mathrm{mmol}^{-1}\right)$, aqueous solution of saturated sodium bicarbonate (1 $\left.\times 4 \mathrm{~mL} \mathrm{mmol}^{-1}\right)$ and brine $\left(1 \times 4 \mathrm{~mL} \mathrm{mmol}^{-1}\right)$. The organic layer was dried over $\mathrm{MgSO}_{4}$, the solvents were removed under reduce pressure and column chromatography (Silica gel) gave the product.

4,4-Diphenylbut-3-en-2-one (4a). Prepared according to the GP4 from phosphordiamidate $2 \mathrm{e}(0.130 \mathrm{~g}, 0.5 \mathrm{mmol})$ and phenylmagnesium chloride $(0.70 \mathrm{~mL}, 1.3 \mathrm{mmol})$. Column chromatography (hexane/AcOEt $\left.9: 1, R_{\mathrm{f}}=0.42\right)$ gave $0.087 \mathrm{~g}(78 \%)$ of the title compound as a yellowish oil. ${ }^{1} \mathrm{H}$ NMR $(300 \mathrm{MHz}$, $\left.\mathrm{CDCl}_{3}\right) \delta$ 7.43-7.39 (m, 3H), 7.36-7.28 (m, 5H), 7.25-7.20 (m, $2 \mathrm{H}), 6.58(\mathrm{~s}, 1 \mathrm{H}), 1.88(\mathrm{~s}, 3 \mathrm{H})$, in accordance with literature. ${ }^{31}$

4,4-Bis(4-chlorophenyl)but-3-en-2-one (4b). Prepared according to the GP4 from phosphordiamidate $2 \mathrm{e}(0.130 \mathrm{~g}, 0.5 \mathrm{mmol})$ and 4-chlorophenylmagnesium chloride $(1.30 \mathrm{~mL}, 1.3 \mathrm{mmol})$. Column chromatography (hexane/AcOEt $9: 1, R_{\mathrm{f}}=0.24$ ) gave $0.116 \mathrm{~g}(80 \%)$ of the title compound as a yellowish oil. ${ }^{1} \mathrm{H}$ NMR $\left(400 \mathrm{MHz}, \mathrm{CDCl}_{3}\right) \delta 7.40-7.38(\mathrm{~m}, 2 \mathrm{H}), 7.32-7.30(\mathrm{~m}, 2 \mathrm{H}), 7.21-$ 7.19 (m, 2H), 7.15-7.12 (m, 2H), $6.56(\mathrm{~s}, 1 \mathrm{H}), 1.99(\mathrm{~s}, 3 \mathrm{H}) ;{ }^{13} \mathrm{C}$ NMR (101 MHz, $\left.\mathrm{CDCl}_{3}\right) \delta 199.3,151.5,139.1,137.0,136.0,135.2$, 131.1, 129.8, 129.0, 128.9, 127.7, 31.0; HRMS (APCI) $m / z$ : calcd for $\mathrm{C}_{16} \mathrm{H}_{12} \mathrm{Cl}_{2} \mathrm{O}_{1}[\mathrm{M}+\mathrm{H}]^{+}$291.0338; found 291.0329.

4,4-Bis(4-fluorophenyl)but-3-en-2-one (4c). Prepared according to the GP4 from phosphordiamidate $2 \mathrm{e}(0.130 \mathrm{~g}, 0.5 \mathrm{mmol})$ and 4-fluorophenylmagnesium chloride $(1.30 \mathrm{~mL}, 1.3 \mathrm{mmol})$. Column chromatography (hexane/AcOEt $9: 1, R_{\mathrm{f}}=0.33$ ) gave $0.105 \mathrm{~g}(81 \%)$ of the title compound as a yellowish oil. ${ }^{1} \mathrm{H}$ NMR $\left(400 \mathrm{MHz}, \mathrm{CDCl}_{3}\right) \delta 7.28-7.24(\mathrm{~m}, 2 \mathrm{H}), 7.20-7.17(\mathrm{~m}, 2 \mathrm{H}), 7.13-$ 7.09 (m, 2H), 7.05-7.01 (m, 2H), $6.53(\mathrm{~s}, 1 \mathrm{H}), 1.96(\mathrm{~s}, 3 \mathrm{H}) ;{ }^{13} \mathrm{C}$ NMR (101 MHz, $\left.\mathrm{CDCl}_{3}\right) \delta 199.5,163.6$ (d, $\left.J=251.5 \mathrm{~Hz}\right), 163.1$ (d, $J=250.5 \mathrm{~Hz}), 151.8,136.8(\mathrm{~d}, J=3.1 \mathrm{~Hz}), 134.6(\mathrm{~d}, J=3.5 \mathrm{~Hz})$, $131.5(\mathrm{~d}, J=8.1 \mathrm{~Hz}), 130.4(\mathrm{~d}, J=9.1 \mathrm{~Hz}), 127.2(\mathrm{~d}, J=6.1 \mathrm{~Hz})$, $115.7(\mathrm{~d}, J=5.1 \mathrm{~Hz}), 115.5$ (d, $J=6.1 \mathrm{~Hz}$ ), 30.6; HRMS (APCI) $\mathrm{m} /$ $z$ : calcd for $\mathrm{C}_{16} \mathrm{H}_{12} \mathrm{~F}_{2} \mathrm{O}_{1}[\mathrm{M}+\mathrm{H}]^{+}$259.0929; found 259.0929 .

4,4-Bis(3-methoxyphenyl)but-3-en-2-one (4d). Prepared according to the GP4 from phosphordiamidate $2 \mathrm{e}(0.130 \mathrm{~g}, 0.5 \mathrm{mmol})$ and 3-methoxyphenylmagnesium bromide (1.30 mL, $1.3 \mathrm{mmol})$. Column chromatography (hexane/AcOEt $6: 1, R_{\mathrm{f}}=0.32$ ) gave $0.112 \mathrm{~g}(79 \%)$ of the title compound as a yellowish oil. ${ }^{1} \mathrm{H}$ NMR $\left(400 \mathrm{MHz}, \mathrm{CDCl}_{3}\right) \delta 7.34-7.30(\mathrm{~m}, 1 \mathrm{H}), 7.26-7.22(\mathrm{~m}, 1 \mathrm{H}), 6.97-$ $6.80(\mathrm{~m}, 5 \mathrm{H}), 6.75-6.74(\mathrm{~m}, 1 \mathrm{H}), 6.56(\mathrm{~s}, 1 \mathrm{H}), 3.80(\mathrm{~s}, 3 \mathrm{H}), 3.78(\mathrm{~s}$, $3 \mathrm{H}), 1.89$ (s, 3H); ${ }^{13} \mathrm{C} \mathrm{NMR}\left(101 \mathrm{MHz}, \mathrm{CDCl}_{3}\right) \delta 200.4,159.6$, 159.5 , 153.5, 142.0, 140.2, 129.5, 129.4, 128.0, 122.1, 121.0,
115.0, 114.9, 114.4, 114.0, 55.3, 30.2; HRMS (APCI) $m / z$ : calcd for $\mathrm{C}_{18} \mathrm{H}_{18} \mathrm{O}_{3}[\mathrm{M}+\mathrm{H}]^{+}$283.1329; found 283.1324.

4,4-Bis(3-(methylthio)phenyl)but-3-en-2-one (4e). Prepared according to the GP4 from phosphordiamidate $2 \mathrm{e}(0.130 \mathrm{~g}, 0.5$ $\mathrm{mmol}$ ) and (3-(methylthio)phenyl)magnesium bromide (2.60 $\mathrm{mL}, 1.3 \mathrm{mmol}$ ). Column chromatography (hexane/AcOEt $6: 1$, $\left.R_{\mathrm{f}}=0.34\right)$ gave $0.126 \mathrm{~g}(80 \%)$ of the title compound as a yellowish oil. ${ }^{1} \mathrm{H} \mathrm{NMR}\left(400 \mathrm{MHz}, \mathrm{CDCl}_{3}\right) \delta 7.35-7.24(\mathrm{~m}, 3 \mathrm{H})$, 7.19-7.18 (m, 1H), 7.07-7.06 (m, 1H), 7.03-6.96 (m, 3H), 6.55 (s, 1H), 2.47 (s, 3H), 2.45 (s, 3H), $1.92(\mathrm{~s}, 3 \mathrm{H}) ;{ }^{13} \mathrm{C}$ NMR (101 MHz, $\left.\mathrm{CDCl}_{3}\right) \delta 200.1,152.9,141.1,139.2,139.2,139.1,128.9,128.8$, 128.2, 127.4, 127.1, 126.7, 126.2, 126.1, 125.3, 30.4, 15.7, 15.6; HRMS (APCI) $m / z$ : calcd for $\mathrm{C}_{18} \mathrm{H}_{18} \mathrm{OS}_{2}[\mathrm{M}+\mathrm{H}]^{+} 315.0872$; found 315.0879 .

4,4-Di-o-tolylbut-3-en-2-one (4f). Prepared according to the GP4 from phosphordiamidate $2 \mathrm{e}(0.130 \mathrm{~g}, 0.5 \mathrm{mmol})$ and $o$ tolylmagnesium chloride $(1.30 \mathrm{~mL}, 1.3 \mathrm{mmol})$. Column chromatography (hexane/AcOEt $\left.6: 1, R_{\mathrm{f}}=0.49\right)$ gave $0.094 \mathrm{~g}(75 \%)$ of the title compound as a yellowish solid, $\mathrm{mp}=62.0-65.0^{\circ} \mathrm{C}$. ${ }^{1} \mathrm{H}$ NMR $\left(400 \mathrm{MHz}, \mathrm{CDCl}_{3}\right) \delta 7.28-7.03(\mathrm{~m}, 8 \mathrm{H}), 6.31(\mathrm{~s}, 1 \mathrm{H}), 2.35$ (s, 3H), $2.10(\mathrm{~s}, 3 \mathrm{H}), 1.85(\mathrm{~s}, 3 \mathrm{H}) ;{ }^{13} \mathrm{C} \mathrm{NMR}\left(101 \mathrm{MHz}, \mathrm{CDCl}_{3}\right)$ $\delta$ 200.2, 153.8, 140.3, 139.4, 135.7, 135.6, 132.3, 131.3, 130.6, 130.1, 129.9, 128.7, 128.4, 125.8, 125.8, 29.9, 21.0, 19.9; HRMS (APCI) $m / z$ : calcd for $\mathrm{C}_{18} \mathrm{H}_{18} \mathrm{O}[\mathrm{M}+\mathrm{H}]^{+}$251.1430; found 251.1432.

4-Octyldodec-3-en-2-one (4g). Prepared according to the GP4 from phosphordiamidate $2 \mathrm{e}(0.13 \mathrm{~g}, 0.5 \mathrm{mmol})$ and octylmagnesium chloride $(0.6 \mathrm{~mL}, 1.3 \mathrm{mmol})$. Column chromatography (hexane/AcOEt $\left.6: 1, R_{\mathrm{f}}=0.41\right)$ gave $0.124 \mathrm{~g}(84 \%)$ of the title compound as a yellowish oil. ${ }^{1} \mathrm{H} \mathrm{NMR}\left(400 \mathrm{MHz}, \mathrm{CDCl}_{3}\right) \delta 6.01$ (s, 1H), 2.55-2.51 (m, 2H), $2.16(\mathrm{~s}, 3 \mathrm{H}), 2.12-2.08(\mathrm{~m}, 2 \mathrm{H}), 1.46-$ $1.25(\mathrm{~m}, 24 \mathrm{H}), 0.90-0.85(\mathrm{~m}, 6 \mathrm{H}) ;{ }^{13} \mathrm{C} \mathrm{NMR}$ (101 MHz, $\mathrm{CDCl}_{3}$ ) $\delta 198.4,163.8,122.9,38.7,32.4,31.92,31.88,31.85$, 30.0, 29.44, 29.42, 29.3, 29.2, 28.6, 27.8, 22.67, 22.66, 14.12, 14.11; HRMS (APCI) $m / z$ : calcd for $\mathrm{C}_{20} \mathrm{H}_{38} \mathrm{O}[\mathrm{M}+\mathrm{H}]^{+}$295.2995; found 295.2984.

1,1-Diphenylhex-1-en-3-one (4h). Prepared according to the GP4 from phosphordiamidate $2 \mathrm{f}(0.149 \mathrm{~g} ; 0.51 \mathrm{mmol})$ and phenylmagnesium chloride $(0.65 \mathrm{~mL}, 1.28 \mathrm{mmol})$. Column chromatography (hexane/AcOEt $20: 1, R_{\mathrm{f}}=0.23$ ) gave $0.101 \mathrm{~g}$ (79\%) of the title compound as a yellowish oil. ${ }^{1} \mathrm{H}$ NMR $(300$ $\left.\mathrm{MHz} \mathrm{CDCl}_{3}\right) \delta 7.41-7.29(\mathrm{~m}, 8 \mathrm{H}), 7.21-7.18(\mathrm{~m}, 2 \mathrm{H}), 6.58(\mathrm{~s}$, $1 \mathrm{H}), 2.22(\mathrm{t}, J=7.3 \mathrm{~Hz}, 2 \mathrm{H}), 1.59-1.46(\mathrm{~m}, 2 \mathrm{H}), 0.79(\mathrm{t}, J=7.4 \mathrm{~Hz}$, $3 \mathrm{H})$, in accordance with literature. ${ }^{32}$

1,1-Bis(4-chlorophenyl)hex-1-en-3-one (4i). Prepared according to the GP4 from phosphordiamidate $2 \mathrm{f}(0.146 \mathrm{~g} ; 0.50 \mathrm{mmol})$ and 4-chlorophenylmagnesium chloride $(1.30 \mathrm{~mL}, 1.25 \mathrm{mmol})$. Column chromatography (hexane/AcOEt $20: 1, R_{\mathrm{f}}=0.31$ ) gave $0.121 \mathrm{~g}(76 \%)$ of the title compound as a yellowish oil. ${ }^{1} \mathrm{H}$ NMR (300 MHz, $\left.\mathrm{CDCl}_{3}\right) \delta 7.37-7.35(\mathrm{~m}, 2 \mathrm{H}), 7.32-7.29(\mathrm{~m}, 2 \mathrm{H}), 7.21-$ $7.19(\mathrm{~m}, 2 \mathrm{H}), 7.12-7.10(\mathrm{~m}, 2 \mathrm{H}), 6.57(\mathrm{~s}, 1 \mathrm{H}), 2.33(\mathrm{t}, J=7.3 \mathrm{~Hz}$, $2 \mathrm{H}), 1.61-1.51(\mathrm{~m}, 2 \mathrm{H}), 0.85(\mathrm{t}, J=7.4 \mathrm{~Hz}, 3 \mathrm{H}) ;{ }^{13} \mathrm{C}$ NMR $(100$ $\left.\mathrm{MHz}, \mathrm{CDCl}_{3}\right) \delta 201.2,150.8,139.1,136.9,135.6,134.7,130.8$, 129.6, 128.7, 128.6, 126.4, 45.6, 17.6, 13.7; HRMS (APCI) m/z: calcd for $\mathrm{C}_{18} \mathrm{H}_{16} \mathrm{Cl}_{2} \mathrm{O}[\mathrm{M}+\mathrm{H}]^{+}$319.0651; found 319.0650.

1,1-Bis(4-fluorophenyl)hex-1-en-3-one (4j). Prepared according to the GP4 from phosphordiamidate $2 \mathrm{f}(0.146 \mathrm{~g} ; 0.50 \mathrm{mmol})$ and 
4-fluorophenylmagnesium bromide (1.30 mL, $1.25 \mathrm{mmol})$. Column chromatography (hexane/AcOEt $20: 1, R_{\mathrm{f}}=0.24$ ) gave $0.110 \mathrm{~g}(77 \%)$ of the title compound as a yellowish oil. ${ }^{1} \mathrm{H}$ NMR $\left(300 \mathrm{MHz}, \mathrm{CDCl}_{3}\right) \delta$ 7.27-7.24 (m, 2H), 7.18-7.14 (m, 2H), 7.10$7.00(\mathrm{~m}, 4 \mathrm{H}), 6.53(\mathrm{~s}, 1 \mathrm{H}), 2.30(\mathrm{t}, J=7.3 \mathrm{~Hz}, 2 \mathrm{H}), 1.60-1.51(\mathrm{~m}$, $2 \mathrm{H}), 0.84(\mathrm{t}, J=7.3 \mathrm{~Hz}, 3 \mathrm{H}) ;{ }^{13} \mathrm{C} \mathrm{NMR}\left(100 \mathrm{MHz}, \mathrm{CDCl}_{3}\right) \delta 201.5$, $163.5(\mathrm{~d}, J=250.4 \mathrm{~Hz}), 162.9(\mathrm{~d}, J=248.6 \mathrm{~Hz}) 151.2,137.1(\mathrm{~d}, J=$ $3.5 \mathrm{~Hz}), 134.6$ (d, $J=3.5 \mathrm{~Hz}), 131.3(\mathrm{~d}, J=8.3 \mathrm{~Hz}), 130.2(\mathrm{~d}, J=$ $8.4 \mathrm{~Hz}), 126.1,115.5(\mathrm{~d}, J=10.9 \mathrm{~Hz}), 115.3(\mathrm{~d}, J=10.8 \mathrm{~Hz}), 45.5$, 17.6, 13.7; HRMS (APCI) $m / z$ : calcd for $\mathrm{C}_{18} \mathrm{H}_{16} \mathrm{~F}_{2} \mathrm{O}[\mathrm{M}+\mathrm{H}]^{+}$ 287.1242; found 287.1243.

1,1-Bis(3-methoxyphenyl)hex-1-en-3-one (4k). Prepared according to the GP4 from phosphordiamidate $2 \mathrm{f}$ (0.146 g; 0.50 $\mathrm{mmol})$ and 3-methoxyphenylmagnesium bromide $(1.30 \mathrm{~mL}$, $1.25 \mathrm{mmol}$ ). Column chromatography (hexane/AcOEt $20: 1, R_{\mathrm{f}}$ $=0.18$ ) gave $0.129 \mathrm{~g}(83 \%)$ of the title compound as a yellowish oil. ${ }^{1} \mathrm{H}$ NMR (300 MHz, $\left.\mathrm{CDCl}_{3}\right) \delta 7.32-7.22(\mathrm{~m}, 2 \mathrm{H}), 6.95-6.71$ $(\mathrm{m}, 6 \mathrm{H}), 6.55(\mathrm{~s}, 1 \mathrm{H}), 3.78(\mathrm{~s}, 3 \mathrm{H}), 3.77(\mathrm{~s}, 3 \mathrm{H}), 2.21(\mathrm{t}, J=7.3 \mathrm{~Hz}$, 2H), 1.56-1.47 (m, 2H), $0.79(\mathrm{t}, J=7.3 \mathrm{~Hz}, 3 \mathrm{H}) ;{ }^{13} \mathrm{C}$ NMR (75 $\left.\mathrm{MHz}, \mathrm{CDCl}_{3}\right) \delta 202.6,159.4,159.4,152.4,142.2,140.2,129.3$, 127.0, 121.9, 120.9, 114.9, 114.5, 114.1, 114.0, 55.3, 55.2, 44.9, 17.8, 13.7; HRMS (APCI) $m / z$ : calcd for $\mathrm{C}_{20} \mathrm{H}_{22} \mathrm{O}_{3}[\mathrm{M}+\mathrm{H}]^{+}$ 311.1642; found 311.1645.

1,1-Bis(3-(methylthio)phenyl)hex-1-en-3-one (4l). Prepared according to the GP4 from phosphordiamidate $2 f(0.146 \mathrm{~g} ; 0.50$ $\mathrm{mmol}$ ) and (3-(methylthio)phenyl)magnesium bromide (1.40 $\mathrm{mL}, 1.25 \mathrm{mmol}$ ). Column chromatography (hexane/AcOEt $\left.30: 1, R_{\mathrm{f}}=0.11\right)$ gave $0.122 \mathrm{~g}(71 \%)$ of the title compound as a yellowish oil. ${ }^{1} \mathrm{H}$ NMR $\left(300 \mathrm{MHz}, \mathrm{CDCl}_{3}\right) \delta 7.30-7.18(\mathrm{~m}, 5 \mathrm{H})$, 7.05-6.94 (m, 3H), $6.54(\mathrm{~s}, 1 \mathrm{H}), 2.46(\mathrm{~s}, 3 \mathrm{H}), 2.46(\mathrm{~s}, 3 \mathrm{H}), 2.45(\mathrm{~s}$, $3 \mathrm{H}), 2.24(\mathrm{t}, J=7.3 \mathrm{~Hz}, 2 \mathrm{H}), 1.57-1.48(\mathrm{~m}, 2 \mathrm{H}), 0.81(\mathrm{t}, J=7.4 \mathrm{~Hz}$, $3 \mathrm{H}) ;{ }^{13} \mathrm{C} \mathrm{NMR}\left(75 \mathrm{MHz}, \mathrm{CDCl}_{3}\right.$ ) $\delta$ 202.2, 151.9, 141.4, 139.3, 138.9, 138.8, 128.8, 128.6, 127.2, 127.16, 127.13, 126.5, 126.2, 126.1, 125.2 , 45.1, 17.7, 15.7, 15.7, 13.7; HRMS (APCI) $m / z$ : calcd for $\mathrm{C}_{20} \mathrm{H}_{22} \mathrm{OS}_{2}[\mathrm{M}+\mathrm{H}]^{+}$343.1185; found 343.1188.

1,1-Di-o-tolylhex-1-en-3-one (4m). Prepared according to the GP4 from phosphordiamidate $2 f(0.146 \mathrm{~g} ; 0.50 \mathrm{mmol})$ and $o$ tolylmagnesium chloride $(1.30 \mathrm{~mL}, 1.25 \mathrm{mmol})$. Column chromatography (hexane/AcOEt $30: 1, R_{\mathrm{f}}=0.28$ ) gave $0.103 \mathrm{~g}(74 \%)$ of the title compound as a yellowish oil. ${ }^{1} \mathrm{H}$ NMR $(300 \mathrm{MHz}$, $\left.\mathrm{CDCl}_{3}\right) \delta 7.26-7.06(\mathrm{~m}, 8 \mathrm{H}), 6.33(\mathrm{~s}, 1 \mathrm{H}), 2.33(\mathrm{~s}, 3 \mathrm{H}), 2.19(\mathrm{t}, J=$ $7.3 \mathrm{~Hz}, 2 \mathrm{H}), 2.10(\mathrm{~s}, 3 \mathrm{H}), 1.54-1.49(\mathrm{~m}, 2 \mathrm{H}), 0.79(\mathrm{t}, J=7.4 \mathrm{~Hz}$, $3 \mathrm{H}) ;{ }^{13} \mathrm{C}$ NMR $\left(75 \mathrm{MHz}, \mathrm{CDCl}_{3}\right) \delta 202.3,152.7,140.7,139.5$, $135.7,135.6$, 131.2, 131.1, 130.4, 129.8, 129.8, 128.3, 128.2, 125.7, 125.6, 44.8, 20.9, 19.9, 17.7, 13.7; HRMS (APCI) $m / z$ : calcd for $\mathrm{C}_{20} \mathrm{H}_{22} \mathrm{O}[\mathrm{M}+\mathrm{H}]^{+} 279.1743$; found 279.1746.

1,1-Di(thiophen-2-yl)hex-1-en-3-one (4n). Prepared according to the GP4 from phosphordiamidate $2 \mathrm{f}(0.146 \mathrm{~g} ; 0.50 \mathrm{mmol})$ and 2-thienylmagnesium bromide $(1.30 \mathrm{~mL}, 1.26 \mathrm{mmol})$. Column chromatography (hexane/AcOEt $20: 1, R_{\mathrm{f}}=0.38$ ) gave $0.098 \mathrm{~g}$ (75\%) of the title compound as a brownish oil. ${ }^{1} \mathrm{H}$ NMR (300 $\left.\mathrm{MHz} \mathrm{CDCl}_{3}\right) \delta$ 7.48-7.46 (m, 1H), 7.38-7.36 (m, 1H), 7.15-7.12 $(\mathrm{m}, 1 \mathrm{H}), 7.10-7.08(\mathrm{~m}, 2 \mathrm{H}), 7.03-7.00(\mathrm{~m}, 1 \mathrm{H}), 6.61(\mathrm{~d}, J=$ $1.7 \mathrm{~Hz}, 1 \mathrm{H}), 2.28(\mathrm{td}, J=7.3,1.8 \mathrm{~Hz}, 2 \mathrm{H}), 1.62-1.49(\mathrm{~m}, 2 \mathrm{H}), 0.83$ $(\mathrm{td}, J=7.4,1.8 \mathrm{~Hz}, 3 \mathrm{H}) ;{ }^{13} \mathrm{C} \mathrm{NMR}\left(75 \mathrm{MHz}, \mathrm{CDCl}_{3}\right) \delta 201.2$, $145.0,138.6$, 138.2, 129.9, 129.6, 128.1, 127.9, 127.7, 126.9,
125.5, 44.9, 17.9, 13.7; HRMS (APCI) $m / z$ : calcd for $\mathrm{C}_{14} \mathrm{H}_{14} \mathrm{OS}_{2}[\mathrm{M}$ $+\mathrm{H}]^{+}$263.0559; found 263.0551.

2-Benzyl-1-phenylhept-2-en-4-one (4o). Prepared according to the GP4 from phosphordiamidate $2 \mathrm{f}(0.146 \mathrm{~g} ; 0.50 \mathrm{mmol})$ and benzylmagnesium chloride $(1.30 \mathrm{~mL}, 1.26 \mathrm{mmol})$. Column chromatography (hexane/AcOEt $50: 1, R_{\mathrm{f}}=0.28$ ) gave $0.088 \mathrm{~g}$ $(63 \%)$ of the title compound as a yellowish oil. ${ }^{1} \mathrm{H}$ NMR (300 $\left.\mathrm{MHz} \mathrm{CDCl}_{3}\right) \delta 7.33-7.22(\mathrm{~m}, 8 \mathrm{H}), 7.12-7.09(\mathrm{~m}, 2 \mathrm{H}), 6.09(\mathrm{~s}$, $1 \mathrm{H}), 3.94(\mathrm{~s}, 2 \mathrm{H}), 3.33(\mathrm{~s}, 2 \mathrm{H}), 2.46(\mathrm{t}, J=7.3 \mathrm{~Hz}, 2 \mathrm{H}), 1.68-1.62$ $(\mathrm{m}, 2 \mathrm{H}), 0.94(\mathrm{t}, J=7.4 \mathrm{~Hz}, 3 \mathrm{H}) ;{ }^{13} \mathrm{C} \mathrm{NMR}\left(100 \mathrm{MHz}, \mathrm{CDCl}_{3}\right)$ $\delta$ 201.3, 157.6, 138.9, 137.8, 129.3, 129.1, 128.5, 128.4, 126.6, 126.2, $125.646 .5,43.5,36.8,17.5,13.8$; HRMS (APCI) $\mathrm{m} / z$ : calcd for $\mathrm{C}_{20} \mathrm{H}_{22} \mathrm{O}[\mathrm{M}+\mathrm{H}]^{+} 279.1743$; found 279.1738.

6-Octyltetradec-5-en-4-one (4p). Prepared according to the GP4 from phosphordiamidate $2 \mathrm{f}(0.148 \mathrm{~g} ; 0.51 \mathrm{mmol})$ and octylmagnesium chloride ( $0.63 \mathrm{~mL}, 1.26 \mathrm{mmol}$ ). Column chromatography (hexane/AcOEt $\left.30: 1, R_{\mathrm{f}}=0.44\right)$ gave $0.147 \mathrm{~g}(90 \%)$ of the title compound as a colorless oil. ${ }^{1} \mathrm{H}$ NMR $\left(300 \mathrm{MHz}, \mathrm{CDCl}_{3}\right)$ $\delta 5.99(\mathrm{~s}, 1 \mathrm{H}), 2.55-2.51(\mathrm{~m}, 2 \mathrm{H}), 2.39(\mathrm{t}, J=7.3 \mathrm{~Hz}, 2 \mathrm{H}), 2.12-$ 2.08 (m, 2H), 1.64-1.58 (m, 2H), 1.44-1.25 (m, 24H), 0.94-0.86 $(\mathrm{m}, 9 \mathrm{H}) ;{ }^{13} \mathrm{C} \mathrm{NMR}\left(75 \mathrm{MHz}, \mathrm{CDCl}_{3}\right) \delta 200.9,163.3,122.6,46.4$, 38.7, 32.5, 31.9, 31.8, 30.0, 29.5, 29.4, 29.4, 29.3, 29.2, 28.7, 27.8, 22.7, 22.6, 17.7, 14.1, 13.8; HRMS (APCI) $m / z$ : calcd for $\mathrm{C}_{22} \mathrm{H}_{42} \mathrm{O}$ $[\mathrm{M}+\mathrm{H}]^{+}$323.3308; found 323.3310.

1,3,3-Triphenylprop-2-en-1-one (4q). Prepared according to the GP4 from phosphordiamidate $2 \mathrm{~g}(0.160 \mathrm{~g} ; 0.50 \mathrm{mmol})$ and phenylmagnesium chloride $(0.70 \mathrm{~mL}, 1.30 \mathrm{mmol})$. Column chromatography (hexane/AcOEt $9: 1, R_{\mathrm{f}}=0.32$ ) gave $0.129 \mathrm{~g}$ $(97 \%)$ of the title compound as a yellow oil. ${ }^{1} \mathrm{H}$ NMR $(400 \mathrm{MHz}$, $\left.\mathrm{CDCl}_{3}\right) \delta$ 7.92-7.90 (m, 2H), 7.48-7.46 (m, 1H), 7.41-7.36 (m, $7 \mathrm{H}), 7.28-7.26(\mathrm{~m}, 3 \mathrm{H}), 7.19-7.12(\mathrm{~m}, 2 \mathrm{H}), 7.12(\mathrm{~s}, 1 \mathrm{H})$, in accordance with literature. ${ }^{33}$

1-Phenyl-3,3-di-o-tolylprop-2-en-1-one (4r). Prepared according to the GP4 from phosphordiamidate $2 \mathrm{~g}(0.160 \mathrm{~g} ; 0.50 \mathrm{mmol})$ and 2-tolylmagnesium chloride $(1.30 \mathrm{~mL}, 1.30 \mathrm{mmol})$. Column chromatography (hexane/AcOEt $9: 1, R_{\mathrm{f}}=0.35$ ) gave $0.144 \mathrm{~g}$ (95\%) of the title compound as a yellow oil. ${ }^{1} \mathrm{H}$ NMR $(400 \mathrm{MHz}$, $\left.\mathrm{CDCl}_{3}\right) \delta 7.90-7.88(\mathrm{~m}, 2 \mathrm{H}), 7.50-7.46(\mathrm{~m}, 1 \mathrm{H}), 7.40-7.36(\mathrm{~m}$, $2 \mathrm{H}), 7.25-7.06(\mathrm{~m}, 8 \mathrm{H}), 6.98(\mathrm{~s}, 1 \mathrm{H}), 2.36(\mathrm{~s}, 3 \mathrm{H}), 2.09(\mathrm{~s}, 3 \mathrm{H}) ;{ }^{13} \mathrm{C}$ $\mathrm{NMR}\left(101 \mathrm{MHz}, \mathrm{CDCl}_{3}\right) \delta 192.0,154.9,141.1,139.6,138.3,135.8$, $135.7,132.6,131.2$, 130.3, 129.9, 129.6, 128.5, 128.4, 128.3, 128.0, 128.0, 125.9, 125.5, 21.0, 20.1; HRMS (APCI) $m / z$ : calcd for $\mathrm{C}_{23} \mathrm{H}_{20} \mathrm{O}[\mathrm{M}+\mathrm{H}]^{+}$313.1587; found 313.1591.

2-(Diphenylmethylene)cyclohexan-1-one (5a). Prepared according to the GP4 from bromobenzene (0.196 g, $1.25 \mathrm{mmol}),{ }^{n} \mathrm{BuLi}$ (0.50 mL, $1.20 \mathrm{mmol}$ ), phosphordiamidate $2 \mathbf{h}$ (0.152 g, 0.50 mmol). Column chromatography (hexane/AcOEt $20: 1, R_{\mathrm{f}}=$ $0.17)$ gave $0.088 \mathrm{~g},(67 \%)$ of the title compound as a white solid, $\mathrm{mp}=130.0-131.0^{\circ} \mathrm{C} .{ }^{1} \mathrm{H}$ NMR $\left(400 \mathrm{MHz}, \mathrm{CDCl}_{3}\right) \delta 7.34-7.22(\mathrm{~m}$, $6 \mathrm{H})$, 7.14-7.11 (m, 2H), 7.06-7.04 (m, 2H), 2.65-2.59 (m, 4H), 2.01-1.96 (m, 2H), 1.83-1.78 (m, 2H); ${ }^{13} \mathrm{C}$ NMR (100 MHz, $\left.\mathrm{CDCl}_{3}\right) \delta 206.9,144.2,142.2,140.8,138.9,129.9,129.0,128.0$, 127.7, 127.3, 45.0, 34.1, 26.6, 26.3; HRMS (APCI) $m / z$ : calcd for $\mathrm{C}_{19} \mathrm{H}_{18} \mathrm{O}[\mathrm{M}+\mathrm{H}]^{+}$263.1430; found 263.1434.

2-(Diphenylmethylene)cyclopentan-1-one (5b). Prepared according to the GP4 from bromobenzene (0.198 g, $1.26 \mathrm{mmol})$, ${ }^{n}$ BuLi (0.50 mL, $\left.1.20 \mathrm{mmol}\right)$, phosphordiamidate 2i (0.138 g, 
$0.50 \mathrm{mmol}$ ). Column chromatography (hexane/AcOEt $20: 1, R_{\mathrm{f}}$ $=0.08)$ gave $0.085 \mathrm{~g}(68 \%)$ of the title compound as a yellow oil. ${ }^{1} \mathrm{H}$ NMR (300 MHz, $\left.\mathrm{CDCl}_{3}\right) \delta 7.34-7.31(\mathrm{~m}, 6 \mathrm{H}), 7.21-7.17(\mathrm{~m}$, $2 \mathrm{H}), 7.14-7.10(\mathrm{~m}, 2 \mathrm{H}), 2.82(\mathrm{t}, J=7.0 \mathrm{~Hz}, 2 \mathrm{H}), 2.38(\mathrm{t}, J=7.7 \mathrm{~Hz}$, $2 \mathrm{H}), 1.98-1.88(\mathrm{~m}, 2 \mathrm{H})$, in accordance with literature. ${ }^{34}$

2-(Bis(4-methoxyphenyl)methylene)cyclopentan-1-one

(5c). Prepared according to the GP4 from 4-bromoanisole $(0.237 \mathrm{~g}$, $1.26 \mathrm{mmol}),{ }^{n} \mathrm{BuLi}(0.50 \mathrm{~mL}, 1.20 \mathrm{mmol})$, phosphordiamidate $2 \mathrm{i}$ $(0.140 \mathrm{~g}, 0.51 \mathrm{mmol})$. Column chromatography (hexane/AcOEt $\left.9: 1, R_{\mathrm{f}}=0.15\right)$ gave $0.115 \mathrm{~g}(74 \%)$ of the title compound as a yellow oil. ${ }^{1} \mathrm{H}$ NMR $\left(400 \mathrm{MHz}, \mathrm{CDCl}_{3}\right) \delta 7.14-7.12(\mathrm{~m}, 2 \mathrm{H})$, 7.06-7.04 (m, 2H), 6.86-6.83 (m, 4H), $3.82(\mathrm{~s}, 6 \mathrm{H}), 2.81(\mathrm{t}, J=$ $7.0 \mathrm{~Hz}, 2 \mathrm{H}), 2.37(\mathrm{t}, J=7.7 \mathrm{~Hz}, 2 \mathrm{H}), 1.91(\mathrm{p}, J=7.3 \mathrm{~Hz}, 2 \mathrm{H}) ;{ }^{13} \mathrm{C}$ NMR $\left(100 \mathrm{MHz} \mathrm{CDCl}_{3}\right) \delta 206.6,159.7,159.4,148.2,134.6,132.6$, 132.5, 131.6, 131.3, 113.2, 113.1, 55.3, 55.1, 40.0, 33.5, 20.7; HRMS (APCI) $m / z$ : calcd for $\mathrm{C}_{20} \mathrm{H}_{20} \mathrm{O}_{3}[\mathrm{M}+\mathrm{H}]^{+}$309.1485; found 309.1489 .

2-(Diphenylmethylene)cycloheptan-1-one $\quad(\mathbf{5 d})$ Prepared according to the GP4 from bromobenzene ( $0.200 \mathrm{~g}, 1.28 \mathrm{mmol})$, ${ }^{n}$ BuLi (0.51 mL, $\left.1.22 \mathrm{mmol}\right)$, phosphordiamidate 2j (0.160 g, $0.52 \mathrm{mmol}$ ). Column chromatography (hexane/AcOEt $20: 1, R_{\mathrm{f}}$ $=0.15)$ gave $0.120 \mathrm{~g}(83 \%)$ of the title compound as a white solid, $\mathrm{mp}=79.5-81.5{ }^{\circ} \mathrm{C} .{ }^{1} \mathrm{H}$ NMR $\left(400 \mathrm{MHz}, \mathrm{CDCl}_{3}\right) \delta 7.37-7.19$ $(\mathrm{m}, 8 \mathrm{H}), 7.11-7.08(\mathrm{~m}, 2 \mathrm{H}), 2.50-2.47(\mathrm{~m}, 2 \mathrm{H}), 2.39-2.36(\mathrm{~m}$, $2 \mathrm{H}), 1.92-1.88(\mathrm{~m}, 2 \mathrm{H}), 1.72-1.61(\mathrm{~m}, 4 \mathrm{H}) ;{ }^{13} \mathrm{C}$ NMR $(100 \mathrm{MHz}$, $\left.\mathrm{CDCl}_{3}\right) \delta 211.2,142.5,141.8,141.5,140.9,128.9,128.6,128.3$, 128.1, 127.22, 127.20, 43.4, 30.3, 29.3, 29.2, 24.2; HRMS (APCI) $m / z$ : calcd for $\mathrm{C}_{20} \mathrm{H}_{20} \mathrm{O}[\mathrm{M}+\mathrm{H}]^{+} 277.1587$; found 277.1586.

2-(Bis(4-methoxyphenyl)methylene)cycloheptan-1-one

(5e). Prepared according to the GP4 from 4-bromoanisole $(0.240 \mathrm{~g}$, $1.28 \mathrm{mmol}),{ }^{n} \mathrm{BuLi}(0.51 \mathrm{~mL}, 1.22 \mathrm{mmol})$, phosphordiamidate $2 \mathrm{j}$ (0.152 g, $0.50 \mathrm{mmol}$ ). Column chromatography (hexane/AcOEt $\left.9: 1, R_{\mathrm{f}}=0.24\right)$ gave $0.152 \mathrm{~g}(90 \%)$ of the title compound as a yellow oil. ${ }^{1} \mathrm{H}$ NMR $\left(400 \mathrm{MHz}, \mathrm{CDCl}_{3}\right) \delta 7.12-7.10(\mathrm{~m}, 2 \mathrm{H})$, 7.00-6.98 (m, 2H), 6.88-6.86 (m, 2H), 6.77-6.74 (m, 2H), 3.81 (s, $3 \mathrm{H}), 3.76(\mathrm{~s}, 3 \mathrm{H}), 2.50-2.41(\mathrm{~m}, 2 \mathrm{H}), 2.40-2.38$ (m, 2H), 1.90-1.86 $(\mathrm{m}, 2 \mathrm{H}), 1.71-1.60(\mathrm{~m}, 4 \mathrm{H}) ;{ }^{13} \mathrm{C} \mathrm{NMR}\left(100 \mathrm{MHz}, \mathrm{CDCl}_{3}\right) \delta 211.7$, $158.7,158.7,141.4,141.4,134.4$, 133.6, 130.4, 13.99, 113.0, 113.4 , 55.19, 55.1, 43.4, 30.5, 29.4, 29.3, 24.3; HRMS (APCI) $m / z$ : calcd for $\mathrm{C}_{22} \mathrm{H}_{24} \mathrm{O}_{3}[\mathrm{M}+\mathrm{H}]^{+}$337.1798; found 337.1802.

2-(Di(thiophen-2-yl)methylene)cycloheptan-1-one (5f). Prepared according to the GP4 from thiophene $(0.104 \mathrm{~g}, 1.23 \mathrm{mmol})$, ${ }^{n}$ BuLi (0.49 mL, $\left.1.17 \mathrm{mmol}\right)$, phosphordiamidate $2 \mathrm{j}(0.156 \mathrm{~g}$, $0.51 \mathrm{mmol}$ ). Column chromatography (hexane/AcOEt $20: 1, R_{\mathrm{f}}$ $=0.28)$ gave $0.059 \mathrm{~g}(40 \%)$ of the title compound as an orange oil. ${ }^{1} \mathrm{H}$ NMR (400 MHz, $\mathrm{CDCl}_{3}$ ) $\delta$ 7.36-7.35 (m, 1H), 7.26-7.24 (m, 1H), 7.04-6.99 (m, 2H), 6.93-6.88 (m, 2H), 2.53-2.46 (m, $4 \mathrm{H}), 1.86-1.67(\mathrm{~m}, 6 \mathrm{H}) ;{ }^{13} \mathrm{C} \mathrm{NMR}\left(100 \mathrm{MHz}, \mathrm{CDCl}_{3}\right) \delta 211.4$, $145.1,142.4$, 141.6, 128.0, 127.8, 126.8, 126.7, 126.4, 126.3, 124.7, 42.7, 30.9, 28.4, 28.3, 23.7; HRMS (APCI) $m / z$ : calcd for $\mathrm{C}_{16} \mathrm{H}_{16} \mathrm{OS}_{2}[\mathrm{M}+\mathrm{H}]^{+}$289.0715; found 289.0719.

3-Methyl-4,4-diphenylbut-3-en-2-one (5g). Prepared according to the GP4 from bromobenzene $(0.20 \mathrm{~g}, 1.3 \mathrm{mmol}),{ }^{n} \mathrm{BuLi}(0.52$ $\mathrm{mL}, 1.25 \mathrm{mmol})$, phosphordiamidate $2 \mathbf{k}(0.140 \mathrm{~g}, 0.50 \mathrm{mmol})$. Column chromatography (hexane/AcOEt $20: 1, R_{\mathrm{f}}=0.37$ ) gave $0.084 \mathrm{~g}(71 \%)$ of the title compound as an yellowish oil. ${ }^{1} \mathrm{H}$ NMR $\left(400 \mathrm{MHz}, \mathrm{CDCl}_{3}\right) \delta$ 7.37-7.26 (m, 6H), 7.18-7.10 (m, 4H), 1.98 (s, 3H), $1.82(\mathrm{~s}, 3 \mathrm{H}) ;{ }^{13} \mathrm{C} \mathrm{NMR}\left(101 \mathrm{MHz}, \mathrm{CDCl}_{3}\right) \delta$ 207.9, 145.1, 141.7, 141.1, 137.7, 129.8, 129.7, 128.4, 128.3, 128.1, 127.7, 30.2, 18.7; HRMS (APCI) $m / z$ : calcd for $\mathrm{C}_{17} \mathrm{H}_{16} \mathrm{O}[\mathrm{M}+\mathrm{H}]^{+}$237.1274; found 237.1266 .

4,4-Bis(4-methoxyphenyl)-3-methylbut-3-en-2-one

(5h).

Prepared according to the GP4 from 4-bromoanisole $(0.240 \mathrm{~g}$, $1.3 \mathrm{mmol}),{ }^{n} \mathrm{BuLi}(0.52 \mathrm{~mL}, 1.25 \mathrm{mmol})$, phosphordiamidate 2k (0.140 g, $0.50 \mathrm{mmol}$ ). Column chromatography (hexane/AcOEt $\left.6: 1, R_{\mathrm{f}}=0.55\right)$ gave $0.072 \mathrm{~g}(49 \%)$ of the title compound as a white solid, $\mathrm{mp}=58.0-61.0{ }^{\circ} \mathrm{C} .{ }^{1} \mathrm{H} \mathrm{NMR}\left(400 \mathrm{MHz}, \mathrm{CDCl}_{3}\right)$ ઈ 7.10-7.07 (m, 2H), 7.02-7.00 (m, 2H), 6.88-6.80 (m, 4H), 3.82 (s, 3H), $3.81(\mathrm{~s}, 3 \mathrm{H}), 2.00(\mathrm{~s}, 3 \mathrm{H}), 1.80(\mathrm{~s}, 3 \mathrm{H}) ;{ }^{13} \mathrm{C}$ NMR (101 $\left.\mathrm{MHz}, \mathrm{CDCl}_{3}\right) \delta$ 207.9, 160.0, 159.1, 145.6, 136.3, 134.6, 133.8, 131.4, 131.4, 113.7, 113.4, 55.3, 30.2, 19.0; HRMS (APCI) $m / z$ : calcd for $\mathrm{C}_{19} \mathrm{H}_{20} \mathrm{O}_{3}[\mathrm{M}+\mathrm{H}]^{+}$297.1485; found 297.1489.

3-Methyl-4,4-di(thiophen-2-yl)but-3-en-2-one (5i). Prepared according to the GP4 from thiophene $(0.110 \mathrm{~g}, 1.30 \mathrm{mmol})$, ${ }^{n} \mathrm{BuLi}(0.52 \mathrm{~mL}, 1.25 \mathrm{mmol})$, phosphordiamidate $2 \mathbf{k}(0.140 \mathrm{~g}$, $0.50 \mathrm{mmol}$ ). Column chromatography (hexane/AcOEt $9: 1, R_{\mathrm{f}}=$ 0.52) gave $0.029 \mathrm{~g}(24 \%)$ of the title compound as a yellow oil. ${ }^{1} \mathrm{H}$ NMR (400 MHz, $\left.\mathrm{CDCl}_{3}\right) \delta$ 7.41-7.39 (m, 2H), 7.06-7.05 (m, 2H), 6.99-6.98 (m, 2H), 2.17 (s, 3H), 1.91 (s, 3H); ${ }^{13} \mathrm{C}$ NMR (101 MHz, $\left.\mathrm{CDCl}_{3}\right) \delta$ 207.2, 143.2, 142.7, 139.8, 130.3, 129.9, 129.5, 128.5, 127.6, 127.2, 127.0, 29.5, 19.3; HRMS (APCI) $\mathrm{m} / \mathrm{z}$ : calcd for $\mathrm{C}_{13} \mathrm{H}_{12} \mathrm{OS}_{2}[\mathrm{M}+\mathrm{H}]^{+}$249.0402; found 249.0406.

\section{Phenyl(3,4,5,6-tetrahydro-[1,1'-biphenyl]-2-yl)methanone} (6). Phenylmagnesium chloride $(0.78 \mathrm{~mL}, 1.53 \mathrm{mmol})$ was added to a mixture of $\mathrm{CuI}(0.029 \mathrm{~g}, 10 \mathrm{~mol} \%)$ in dry ether $(5 \mathrm{~mL})$ cooled to $0{ }^{\circ} \mathrm{C}$. The resultant mixture was stirred for $1 \mathrm{~h}$ at $0{ }^{\circ} \mathrm{C}$ followed by addition of phosporodiamidate $2 \mathrm{~h}(0.156 \mathrm{~g}, 0.51$ $\mathrm{mmol}$ ). Then the mixture was stirred for $3 \mathrm{~h}$ at $23{ }^{\circ} \mathrm{C}$. The crude reaction mixture was quenched with saturated aqueous solution of ammonium chloride $(2 \mathrm{~mL})$, diluted with ether $(30 \mathrm{~mL})$ and the organic layer was washed with water $(30 \mathrm{~mL})$ and brine (30 mL). The organic phase was dried over $\mathrm{MgSO}_{4}$ and the solvents were removed under reduce pressure. Column chromatography (Silica gel, hexane/AcOEt $20: 1, R_{\mathrm{f}}=0.28$ ) gave $0.062 \mathrm{~g}(46 \%)$ of the title compound as a white solid. ${ }^{1} \mathrm{H}$ NMR $\left(300 \mathrm{MHz}, \mathrm{CDCl}_{3}\right) \delta$ 7.69-7.66 (m, 2H), 7.32-7.31 (m, 1H), 7.237.19 (m, 2H), 7.09-6.99 (m, 5H), 2.53-2.45 (m, 4H), 1.91-1.82 $(\mathrm{m}, 4 \mathrm{H})$, in accordance with literature. ${ }^{35}$

(Z)-4-Hydroxy-4,4-diphenylbut-2-en-2-yl bis( $N, N$-dimethylamino)phosphordiamidate (8). Phenylmagnesium chloride (6.58 $\mathrm{mL}, 12.50 \mathrm{mmol}$ ) was added to a solution of phosphordiamidated $2 \mathrm{e}(1.320 \mathrm{~g}, 5.0 \mathrm{mmol})$ cooled to $-78^{\circ} \mathrm{C}$. The resultant reaction mixture was stirred for $2 \mathrm{~h}$ at $23{ }^{\circ} \mathrm{C}$. Then the reaction mixture was quenched with water $(20 \mathrm{~mL})$, the organic layer was separated and the water layer was extracted with ether $(2 \times 40$ $\mathrm{mL})$. Combined organic layers were washed with water $(30 \mathrm{~mL})$, brine $(10 \mathrm{~mL})$, dried over $\mathrm{MgSO}_{4}$ and concentrated under reduce pressure. Column chromatography (Silica gel, hexane/AcOEt $\left.20: 1, R_{\mathrm{f}}=0.53\right)$ gave $1.480 \mathrm{~g}(79 \%)$ of the title compound as a white solid, $\mathrm{mp}=74.0-77.0{ }^{\circ} \mathrm{C} .{ }^{1} \mathrm{H} \mathrm{NMR}\left(500 \mathrm{MHz}, \mathrm{CDCl}_{3}\right)$ $\delta$ 7.59-7.57 (m, 4H), 7.30-7.27 (m, 4H), 7.18-7.15 (m, 2H), 6.22 (s, 1H), $5.74(\mathrm{~s}, 1 \mathrm{H}), 2.42(\mathrm{~d}, J=10.1 \mathrm{~Hz}, 12 \mathrm{H}), 2.05(\mathrm{~s}, 3 \mathrm{H}) ;{ }^{13} \mathrm{C}$ NMR (126 MHz, $\left.\mathrm{CDCl}_{3}\right) \delta 148.8,145.5(\mathrm{~d}, J=7.6 \mathrm{~Hz}), 128.1$, 126.4, 126.2, 122.7 (d, $J=6.6 \mathrm{~Hz}), 75.8,36.2$ (d, $J=4.7 \mathrm{~Hz}), 21.8$; 
${ }^{31} \mathrm{P}$ NMR $\left(202 \mathrm{MHz}, \mathrm{CDCl}_{3}\right) \delta 15.12(\mathrm{~s})$; HRMS (ESI) $\mathrm{m} / \mathrm{z}$ : calcd for $\mathrm{C}_{20} \mathrm{H}_{27} \mathrm{~N}_{2} \mathrm{O}_{3} \mathrm{P}[\mathrm{M}+\mathrm{Na}]^{+}$397.1652; found 397.1660.

\section{Conflicts of interest}

There are no conflicts to declare.

\section{Acknowledgements}

This work was supported from the Grant Agency of the Czech Republic (Grant No 18-12150S).

\section{Notes and references}

1 (a) J. Adrio and J. C. Carretero, ChemCatChem, 2010, 2, 13841386; (b) M. M. Heravi and P. Hajiabbasi, Monatsh. Chem., 2012, 143, 1575-1592; (c) M. M. Heravi, V. Zadsirjan, P. Hajiabbasi and H. Hamidi, Monatsh. Chem., 2019, 150, 535-591.

2 (a) R. Ambre, H. Yang, W.-C. Chen, G. P. A. Yap, T. Jurca and T.-G. Ong, Eur. J. Inorg. Chem., 2019, 3511-3517; (b) F. Fan, J. Tang, M. Luo and X. Zeng, J. Org. Chem., 2018, 83, 13549-13559; (c) P.-P. Chen, E. L. Lucas, M. A. Greene, S.-Q. Zhang, E. J. Tollefson, L. W. Erickson, B. L. H. Taylor, E. R. Jarvo and X. Hong, J. Am. Chem. Soc., 2019, 141, 5835-5855; (d) D. Ghorai, J. Loup, G. Zanoni and L. Ackermann, Synlett, 2019, 30, 429-432.

3 (a) A. Piontek, W. Ochędzan-Siodłak, E. Bisz and M. Szostak, Adv. Synth. Catal., 2019, 361, 2329-2336; (b) Y. Sato, Y. Ashida, D. Yoshitake, M. Hoshino, T. Takemoto and Y. Tanabe, Synthesis, 2018, 50, 4659-4667.

4 C. A. Quesnelle and V. Snieckus, Synthesis, 2018, 50, 43954412.

5 (a) R. Manikandan and M. Jeganmohan, Org. Biomol. Chem., 2015, 13, 10420-10436; (b) E.-i. Negishi, G. Wang, H. Rao and Z. Xu, J. Org. Chem., 2010, 75, 3151-3182; (c) W.-Y. Siau, Y. Zhang and Y. Zhao, in Stereoselective Alkene Synthesis, ed. J. Wang, Springer Berlin Heidelberg, Berlin, Heidelberg, 2012, pp. 33-58, DOI: 10.1007/128_2012_315.

6 (a) M. Drouin, J.-D. Hamel and J.-F. Paquin, Synthesis, 2018, 50, 881-955; (b) M. Maraswami and T.-P. Loh, Synthesis, 2019, 51, 1049-1062; (c) P. Polák, H. Váňová, D. Dvořák and T. Tobrman, Tetrahedron Lett., 2016, 57, 3684-3693.

7 (a) G. Cahiez, V. Habiak and O. Gager, Org. Lett., 2008, 10, 2389-2392; (b) G. Cahiez and H. Avedissian, Synthesis, 1998, 1199-1205; (c) G. Cahiez, O. Gager and V. Habiak, Synthesis, 2008, 2636-2644.

8 (a) D. Fiorito, S. Folliet, Y. Liu and C. Mazet, ACS Catal., 2018, 8, 1392-1398; (b) A. S. E. Karlström, K. Itami and J.-E. Bäckvall, J. Org. Chem., 1999, 64, 1745-1749; (c) C. Sahlberg, A. Quader and A. Claesson, Tetrahedron Lett., 1983, 24, 5137-5138.

9 (a) D. Gauthier, S. Beckendorf, T. M. Gøgsig, A. T. Lindhardt and T. Skrydstrup, J. Org. Chem., 2009, 74, 3536-3539; (b) W. You, Y. Li and M. K. Brown, Org. Lett., 2013, 15, 16101613; (c) J. A. Miller, Tetrahedron Lett., 2002, 43, 7111-7114.
10 (a) P. Knochel, W. Dohle, N. Gommermann, F. F. Kneisel, F. Kopp, T. Korn, I. Sapountzis and V. A. Vu, Angew. Chem., Int. Ed., 2003, 42, 4302-4320; (b) H. Ila, O. Baron, A. J. Wagner and P. Knochel, Chem. Commun., 2006, 583593; (c) R. Li-Yuan Bao, R. Zhao and L. Shi, Chem. Commun., 2015, 51, 6884-6900; (d) C. E. I. Knappke and A. Jacobi von Wangelin, Chem. Soc. Rev., 2011, 40, 49484962.

11 C. C. Silveira, R. B. Guerra and J. V. Comasseto, Tetrahedron Lett., 2007, 48, 5121-5124.

12 R. E. Barrientos-Astigarraga, P. Castelani, C. Y. Sumida, J. Zukerman-Schpector and J. V. Comasseto, Tetrahedron, 2002, 58, 1051-1059.

13 F.-W. Sum and L. Weiler, Can. J. Chem., 1979, 57, 1431-1441. 14 (a) O. J. Yoshitaka, T. Tomofumi and M. Teruaki, Chem. Lett., 2003, 32, 994-995; (b) T. Bach and F. Höfer, J. Org. Chem., 2001, 66, 3427-3434; (c) R. C. D. Brown, C. J. Bataille, R. M. Hughes, A. Kenney and T. J. Luker, J. Org. Chem., 2002, 67, 8079-8085; (d) H. V. Thulasiram, R. M. Phan, S. B. Rivera and C. D. Poulter, J. Org. Chem., 2006, 71, 1739-1741.

15 Selected examples: (a) O. Piva, J. Org. Chem., 1995, 60, 78797883; (b) K. P. Reber, J. Xu and C. A. Guerrero, J. Org. Chem., 2015, 80, 2397-2406; (c) C. C. Nawrat and C. J. Moody, Org. Lett., 2012, 14, 1484-1487; (d) K. Ishigami, S. Yamada and H. Watanabe, Tetrahedron Lett., 2015, 56, 5816-5819.

16 (a) S.-i. Hashimoto, M. Ban, Y. Yanagiya, S. Sakata and S. Ikegami, Tetrahedron Lett., 1991, 32, 4027-4030; (b) D. Das, R. Kant and T. K. Chakraborty, Org. Lett., 2014, 16, 2618-2621.

17 (a) H. M. Cheng, W. Tian, P. A. Peixoto, B. Dhudshia and D. Y.-K. Chen, Angew. Chem., Int. Ed., 2011, 50, 4165-4168; (b) N. Hayashi, K. Yamamoto, N. Minowa, M. Mitomi and M. Nakada, Org. Biomol. Chem., 2010, 8, 1821-1825; (c) N. Hayashi and M. Nakada, Tetrahedron Lett., 2009, 50, 232-235; (d) C. M. Gampe, H. Tsukamoto, T.-S. A. Wang, S. Walker and D. Kahne, Tetrahedron, 2011, 67, 9771-9778.

18 (a) M. Polla and T. Frejd, Tetrahedron, 1993, 49, 2701-2710; (b) L. Pettersson, G. Magnusson, T. Frejd, T. A. Pakkanen, J. C. Négrel, M. Chanon, C. Striley, J. Weidlein, A. Nasiri and Y. Okada, Acta Chem. Scand., 1993, 47, 196-207; (c) V. K. Aggarwal, P. A. Bethel and R. Giles, Chem. Commun., 1999, 325-326.

19 (a) P. Polák and T. Tobrman, Eur. J. Org. Chem., 2019, 957968; (b) P. Polák and T. Tobrman, Org. Biomol. Chem., 2017, 15, 6233-6241; (c) V. Kotek, P. Polák, H. Dvořáková and T. Tobrman, Eur. J. Org. Chem., 2016, 5037-5044; (d) V. Kotek, H. Dvořáková and T. Tobrman, Org. Lett., 2015, 17, 608-611.

20 J. Barluenga, M. Tomás-Gamasa, F. Aznar and C. Valdés, Adv. Synth. Catal., 2010, 352, 3235-3240.

21 Y. Zou, L. Qin, X. Ren, Y. Lu, Y. Li and J. Zhou, Chem.-Eur. J., 2013, 19, 3504-3511.

22 R. Wang and S. Zhang, RSC Adv., 2014, 4, 39497-39507.

23 M. Hossain, U. Das and J. R. Dimmock, Eur. J. Med. Chem., 2019, 183, 111687. 
24 (a) R. C. Boruah and G. S. Nongthombam, Heterocycles, 2019, 98, 19-62; (b) C. C. Meyer, E. Ortiz and M. J. Krische, Chem. Rev., 2020, 120, 3721-3748; (c) J. Lam, K. M. Szkop, E. Mosaferi and D. W. Stephan, Chem. Soc. Rev., 2019, 48, 3592-3612.

25 (a) A. Douchez, A. Geranurimi and W. D. Lubell, Acc. Chem. Res., 2018, 51, 2574-2588; (b) S. Zhang, H. Neumann and M. Beller, Chem. Soc. Rev., 2020, 49, 3187-3210.

26 C. Jiang, H. Lu, W.-H. Xu, J. Wu, T.-Y. Yu, P.-F. Xu and H. Wei, ACS Catal., 2020, 10, 1947-1953.

27 C. Nájera, L. K. Sydnes and M. Yus, Chem. Rev., 2019, 119, 11110-11244.

28 F.-F. Pan, P. Guo, C.-L. Li, P. Su and X.-Z. Shu, Org. Lett., 2019, 21, 3701-3705.
29 (a) Y.-L. An, K. Li, Y. Shen, Z. Hong, L. Chen, Y. Hu, L. Zhou, D. Wang, X. Shi, S. Liu, W. Su, W. Cui, L. Kuai, H. Yang and X. Peng, Org. Lett., 2020, 22, 3931-3935; (b) J. U. Rhee and M. J. Krische, Org. Lett., 2005, 7, 2493-2495.

30 N. Kreutzkamp, J. Pluhatsch and H. Schindler, Arch. Pharm., 1960, 293, 900-907.

31 X. Zeng, Z. Lu, S. Liu, G. B. Hammond and B. Xu, J. Org. Chem., 2017, 82, 13179-13187.

32 Y. Sugawara, W. Yamada, S. Yoshida, T. Ikeno and T. Yamada, J. Am. Chem. Soc., 2007, 129, 12902-12903.

33 L. Yang and Q. Zeng, Synthesis, 2017, 49, 3149-3156.

34 R. C. Larock and C. K. Reddy, Org. Lett., 2000, 2, 3325-3327. 35 Y. Miyahara and Y. N. Ito, J. Org. Chem., 2014, 79, 6801-6807. 